\title{
1 Gene activation by a CRISPR-assistant trans enhancer
}

\section{Xin Hui Xu, Wei Dai, Dan Yang Wang, Jian Wu, Jin Ke Wang*}

3 State Key Laboratory of Bioelectronics, Southeast University, Nanjing 210096, China

$4 \quad$ *To whom correspondence should be addressed:

5 Jinke Wang

6 State Key Laboratory of Bioelectronics,

7 Southeast University, Nanjing 210096, China.

8 Tel.: (86) 025 83793620;

9 Fax: (86) 025 83793620;

10 E-mail: wangjinke@seu.edu.cn 


\section{Abstract}

16 Gene activation is essential to the basic biological research and biomedicine. Therefore, various gene 17 activators such as activation domain-ZNF, TALE and CRISPR proteins have been developed for this end, in 18 which the CRISPR protein dead Cas9 (dCas9) is now most widely used. However, the current gene activators 19 are still limited by their inefficient gene activation activity. In this study, we developed a new strategy, 20 CRISPR-assistant trans enhancer, for activating gene expression in high efficiency by combining dCas921 VP64/sgRNA with a widely used strong enhancer, the CMV enhancer. In this strategy, a trans CMV enhancer 22 DNA was recruited to target gene by dCas9-VP64/sgRNA via annealing between $3^{\prime}$ end of sgRNA and CMV enhancer. The trans enhancer activates gene transcription as the natural looped cis enhancer. The trans

24 enhancer could activate both exogenous reporter gene and variant endogenous genes in various cells, with 25 much higher activation efficiency than the current dCas9 activators.

26 Keywords: CRISPR/dCas9, CMV enhancer, trans enhancer, gene activation 


\section{Introduction}

Artificial activation of gene expression has important role in the basic biological research and biomedical application. For example, gene function is often explored by artificially activating its expression in cells or in vivo in basic research. In biomedicine, cells are often needed to be reprogrammed into induced stem (iPS) cells or other differentiated cells by activating endogenous genes. In medicine, cancers can be treated by inhibiting various kinases, activating genes enhancing immunity (Sagiv-Barfi et al. 2018), apoptosis, and differentiation (McClellan et al. 2015). Therefore, various artificial gene activators have been developed. For instance, the activation domain-fused zinc finger (ZNF), transcription activator-like effector (TALE), and clustered regularly interspaced short palindromic repeats (CRISPR) associated proteins have been developed for gene activation. In these proteins and complexes, the CRISPR-associated (Cas) proteins are now most widely used due to its simplicity (Gao et al. 2014b).

CRISPR is originally an immune system of bacteria to destroy the invaded microphage DNAs by enzymatically digestion. The system has already been developed into a high efficient gene editing tool (Doudna and Charpentier 2014; Mali et al. 2013c). In addition, the system has also been developed as a new kind of gene regulation tool. For example, the dead Cas9 (dCas9) and its associated single guide RNA (sgRNA) have been most widely used to regulate gene expression in recent years (Dominguez et al. 2015; Hilton et al. 2015; Jinek et al. 2012; Kiani et al. 2015; Mali et al. 2013b; Radzisheuskaya et al. 2016; Wang et al. 2016). For this end, both dCas9 and sgRNA have been widely engineered for activating or repressing gene expression. For instance, the dCas9 protein was fused with various gene activation or repression domains, such as VP48 (Cheng et al. 2013), VP160 (Perrin et al. 2017), VP64 (Maeder et al. 2013; Perez-Pinera et al. 2013), VPR (VP64-p65-Rta) (Chavez et al. 2015), and KRAB (Zheng et al. 2018). Additionally, the dCas9 protein was also fused with other functional domains with transcriptional regulatory functions, such as p300(Hilton et al., 2015), LSD1(Kearns et al. 2015), Dnmt3a (Liu et al. 2016a; Saunderson et al. 2017), and Tet1(Choudhury et al. 2016; Liu et al., 2016a). Based on these domains, more elaborate activators have been developed for more potent activation of target genes in mammalian cells, such as SunTag (dcas9-GCN4/sgRNA plus scFV-VP64) (Tanenbaum et al. 2014), and SPH (dCas9GCN4/sgRNA plus scFV-p65-HSF1) (Zhou et al. 2018). More complex, some inducible dCas9 systems have been developed to control activity of dCas9 activators in cells, such as light-activated CRISPR/Cas9 effector (Nihongaki et al. 2015; Polstein and Gersbach 2015), hybrid drug inducible CRISPR/Cas9 technology (HIT) (Lu et al. 2018), and CRISPR activator gated by human antibody-based chemically induced dimerizers (AbCIDs) (Liu et al. 2018). A trouble of these typical dCas9 fusion proteins in their in vivo applications is that they are difficult to be accommodated by adeno-associated virus (AAV), a most suitable type of non-integrating gene therapy vector, due to its limited packaging capacity.

Besides dCas9 engineering, sgRNA has also been engineered to develop new dCas9-based activators. Compared with dCas9 engineering, sgRNA is more simple, flexible and efficient to redesign. Moreover, the engineered sgRNA is more helpful for the in vivo application of dCas9-based activators due to its limited length for virus packaging. The most widely used engineered sgRNA is the sgRNA fused with MS2 loops at the $3^{\prime}$ end (sgRNA-MS aptamer), which can be bound by dimerized MS2 bacteriophage coat proteins fused with transcription-activating domains VP64-HSF1 (MS2-VP64-HSF1, MPH)(Konermann et al. 2015; Liao et al. 2017). The system is now well known as the synergistic activation mediator (SAM) system. Similarly, another engineered sgRNA-based dCas9 activator, named as the Casilio system, was developed, which consists of the dCas9 protein, an sgRNA appended with one or more binding site(s) of RNA-binding protein Pumilio/FBF (PUF) (sgRNA-PBS), and an effector (such as VP64 and p65-HSF1) fused with a PUF domain (PUF fusion) (Cheng et al. 2016). In the same way, by extending sgRNAs to include effector protein 
recruitment sites, the modular scaffold RNAs that encode both target locus and regulatory action were constructed (Zalatan et al. 2015). For these recruitment RNA modules, the well-characterized viral RNA sequences MS2, PP7, and com, which are recognized by the MCP, PCP, and Com RNA-binding proteins, respectively, were used (Zalatan et al., 2015). The transcriptional activation domain VP64 was fused to each of the corresponding RNA-binding proteins (Zalatan et al., 2015). By extending sgRNAs to include modified riboswitches that recognize specific signals, a CRISPR-Cas9-based 'signal conductors' that regulate transcription of endogenous genes in response to external or internal signals of interest (such as drug) was created (Liu et al. 2016b). Clearly, these chimeric sgRNAs limited by their long complexed RNA aptamers and the cognate RNA-binding fusion proteins.

Although variant dCas9-based activators have been developed (Chen and Qi 2017), the current dCas9based transcriptional activators are still inefficient at endogenous gene activation and reprogramming (Gao et al. 2014a). By a systematic comparing of their relative potency and effectiveness across various cell types and species (human, mouse, and fly) (Chavez et al. 2016), it was found that the majority of secondgeneration activators showed improved levels of activation as compared to those of dCas9-VP64, in which three activators including VPR, SAM, and Suntag were most potent. Across a range of target genes and cellular environments, the VPR, SAM, and Suntag systems are consistently superior to the previous VP64 standard. Moreover, VPR, SAM, and Suntag generally fall within an order of magnitude of each other with regard to fold increase in gene expression. More importantly, attempts to build an improved chimeric activator by fusing elements from VPR, SAM, and Suntag were unsuccessful (Chavez et al., 2016). Therefore, future efforts to improve dCas9-based activators may benefit from exploring other unique architectures or novel activation domains.

Almost three decades ago, the human cytomegalovirus (CMV) enhancer/promoter (referred to as CMV enhancer hereafter) was found as a natural mammalian promoter with high transcriptional activity (Boshart et al. 1985). The late studies gradually found that the CMV enhancer is the known strongest promoter in various mammalian cells (Boshart et al., 1985; Foecking and Hofstetter 1986; Ho et al. 2015; Kim et al. 1990). Therefore, this enhancer has been widely used to drive the ectopic expression of various genes in wide range of mammalian cells. For example, the CMV enhancer is also used to drive the ectopic expressions of exogenous genes in broad tissues in transgenic animals (Furth et al. 1991; Schmidt et al. 1990), protein production by gene engineering, and human gene therapy. We have recently further improved the transcriptional activity of the CMV enhancer by changing the natural NF- $\mathrm{KB}$ binding sites in this enhancer into artificially selected NF- $\mathrm{KB}$ binding sequences with high binding affinity (Wang et al. 2018). Therefore, we conceived that an unique architecture may be constructed to further improve dCas9-based activators by using the CMV enhancer.

In this study, mimicking the natural enhancer activating gene expression by a loop structure (Carter $\boldsymbol{e}$ al. 2002; Tolhuis et al. 2002), we developed a new dCas9-based activator by combining dCas9/sgRNA with CMV enhancer. The $3^{\prime}$ end of sgRNA was redesigned to add a short capture sequence in complementary with a stick-end of a double-stranded CMV enhancer. The CMV enhancer is anchored to the promoter region of target gene by dCas9/sgRNA. The dCas9/sgRNA-recruited CMV enhancer thus functions like a natural looped cis enhancer in trans form. We found that the new activator could efficiently activate multiple genes in 6 kinds of cells including 293T, HepG2, PANC-1, HeLa, A549, and HT29. Moreover, the new activator activated expression of transcription factors $H N F 4 \alpha$ in HepG2 cells and E47 in PANC-1 cells could lead to differentiation of these cancer cells.

\section{Results}



in Figure 1a. We modified sgRNA by adding a capture sequence to the $3^{\prime}$ end of normal sgRNA sequence, which produces a $3^{\prime}$ end-extended sgRNA. Because the newly designed sgRNA will be used to capture a trans enhancer DNA, we named it as capture sgRNA (csgRNA). Correspondingly, a linear double-stranded CMV enhancer sequence with a free single-strand $3^{\prime}$ end was designed; we named the CMV fragment as stick CMV (sCMV), which can interact with csgRNA by annealing to the $3^{\prime}$ capture sequence of csgRNA via its stick end. In our conception, with these csgRNA and sCMV designing, when the csgRNA directs the dCas9 protein to the target site that locates in the promoter region of interested gene, the sCMV will be captured to the gDNA-bound dCas9/csgRNA. This interaction process will anchor a sCMV to the promoter region of an interested gene. The anchored SCMV may thus activate the transcription of an interested gene like natural looped cis enhancer. Because the dCas9/csgRNA-anchored sCMV functions like transcription factors in trans, it can be regarded as trans enhancer, in contrast with natural enhancer that functions in cis.

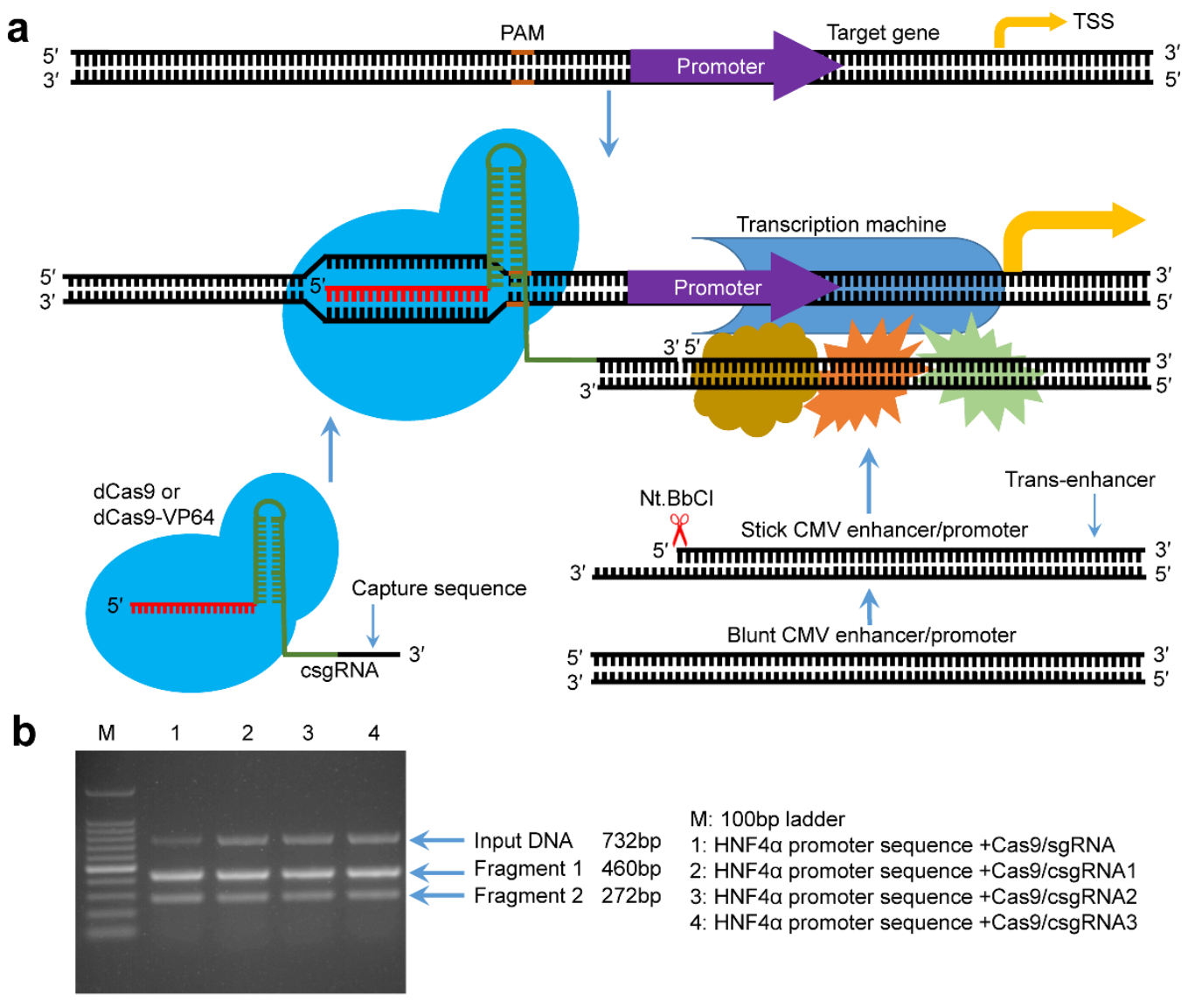

130 Figure 1. Principle of gene expression activation by the CRISPR-assistant trans enhancer and evaluation of designed csgRNAs. a. Schematic illustration of the principle of gene expression activation by the CRISPRassistant trans enhancer. A capture sequence was added at the 3' end of sgRNA, which is used to capture a trans CMV enhancer with a single-stranded overhang that can anneal with the capture sequence of sgRNA. The captured trans CMV enhancer may function like the natural looped cis enhancer to activate transcription of interested gene, including exogenous and endogenous genes. b. In vitro target DNA cutting by the Cas9csgRNA complex. A 732-bp DNA fragment amplified from the HNF4 $\alpha$ promoter region were respectively cut by the Cas $9 / \operatorname{csgRNA}$ and Cas $9 /$ sgRNA complexes. 

enhancer fragment. To find whether the added capture sequence affects the function of sgRNA, we synthesized a normal sgRNA and three csgRNAs targeting to a same site of $H N F 4 \alpha$ promoter by in vitro transcription. Three $\operatorname{csgRNAs}$ had different capture sequences. We used these sgRNAs to associate with the Cas9 endonuclease to cut a 732-bp HNF4 $\alpha$ promoter fragment. The results indicate that the target DNA could be digested by all sgRNAs, including three csgRNAs with variant capture sequences (Figure 1b). This indicates that the capture sequence exerts no effect on the sgRNA function.

\section{Activation of an exogenous reporter gene by trans enhancer}

To find whether the CRISPR-assistant trans enhancer is able to activate gene expression, we constructed a reporter construct of $H N F 4 \alpha$ promoter, pEZX-HP-ZsGreen. We then transfected the 293T cells with sCMV, dCas9, and $\operatorname{csgRNA} 2$. We found that the system activated the expression of reporter gene ZsGreen (Figure 2; dCas9/csgRNA2-sCMV). The activation level is similar to that of dCas9-VP64/sgRNA. Because we used a dCas9 that has no fused transcriptional activation domain like VP64 in the trial transfection, this indicates that the sCMV can not only interact with the dCas9/csgRNA in cell as we conceived, but also function as a transcription factor to activate gene expression as we expected. However, the activation level of dCas9/csgRNA \& sCMV system is far below the activation strength of cis CMV enhancer.

To improve the transcriptional activation performance of dCas9/csgRNA \& sCMV system, we then transfected the 293 T cells with a dCas9-VP64/csgRNA2 \& sCMV system. As a result, we found that the system greatly activated the expression of reporter gene (Figure 2; dCas9-VP64/csgRNA2-sCMV). As a control, the transfection with dCas9-VP64/csgRNA2 \& blunt CMV (bCMV) system obtained an activation level similar to that of dCas9-VP64/sgRNA (Figure 2), indicating that the sCMV genuinely contributed to the gene expression activation. More importantly, we found that the VP64 fused to the dCas9 protein could significantly improve the function of sCMV, possibly by addition or synergy. We then transfected the 293T cells with other two csgRNAs, csgRNA1 and csgRNA3, together with dCas9-VP64 \& sCMV. We found that these two csgRNAs also obtained high level activation (Figure 2), despite inferior to csgRNA2. The transcriptional activation function of trans SCMV was also confirmed by another control transfection, dCas9/csgRNA2 \& bCMV, which showed no transcriptional activation (Figure 2).

To confirm the function of trans enhancer, we also performed more negative control transfections in 293T cells, including the sole dCas9-VP64, sgRNA, and pEZX-HP-ZsGreen, and dCas9-VP64 plus pEZX-HPZsGreen. These transfections all didn't activate the reporter gene expression (Supplementary Figure 1). These data verify that the trans enhancer sCMV can play transcriptional activation role by combination with dCas9/csgRNA. Moreover, the typical transcription activation domain VP64 fused to dCas9 can further improve the transcriptional activation performance of trans enhancer SCMV. Therefore, we adopted the 


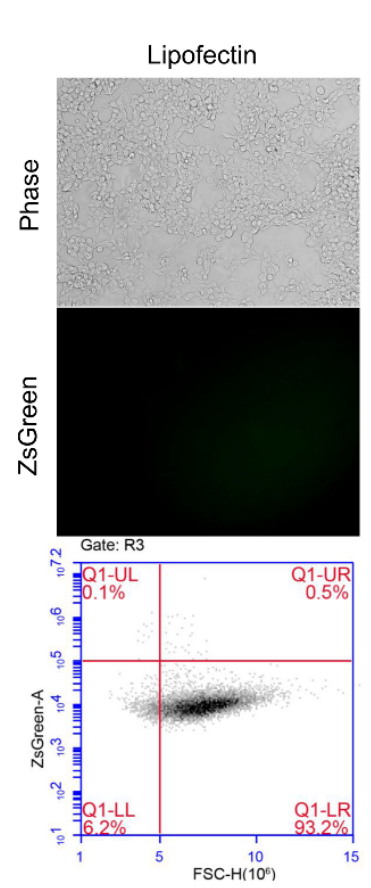

dCas9-VP64/csgRNA1-sCMV
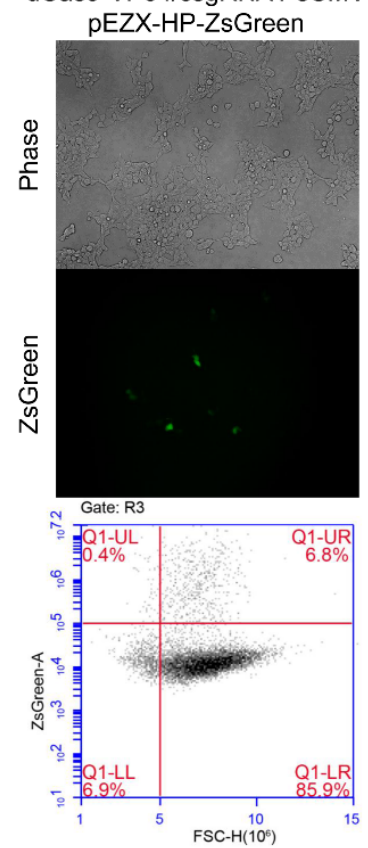

dCas9-VP64/sgRNA

pEZX-HP-ZsGreen
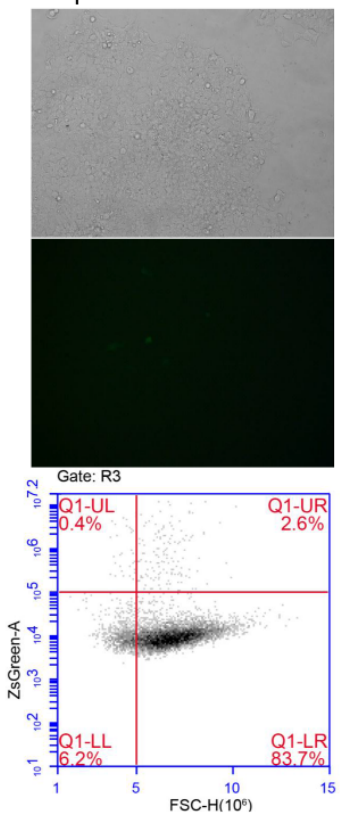

dCas9-VP64/csgRNA2-sCMV pEZX-HP-ZsGreen
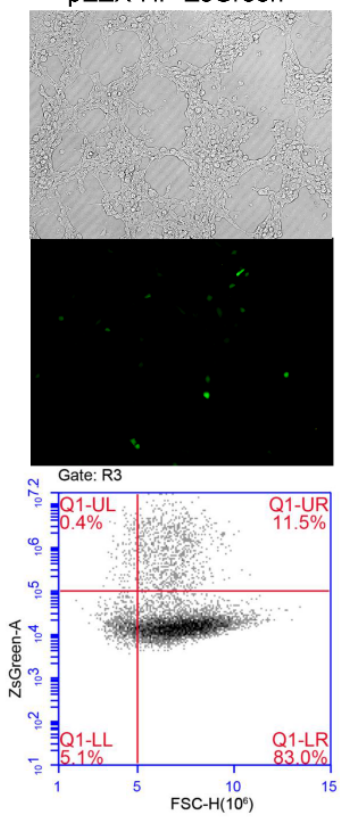

dCas9/csgRNA2-sCMV pEZX-HP-ZsGreen
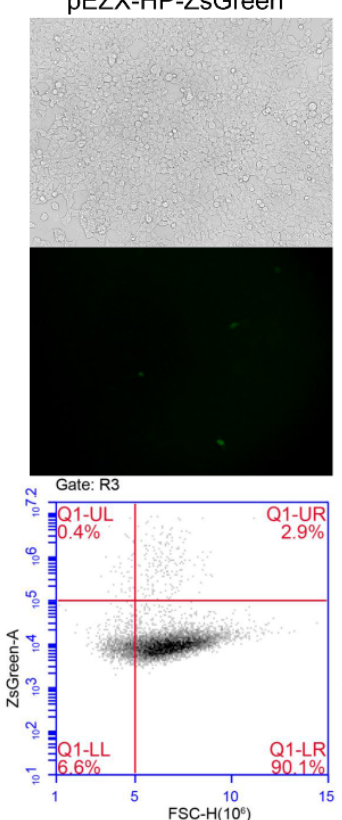

dCas9-VP64/csgRNA3-sCMV pEZX-HP-ZsGreen
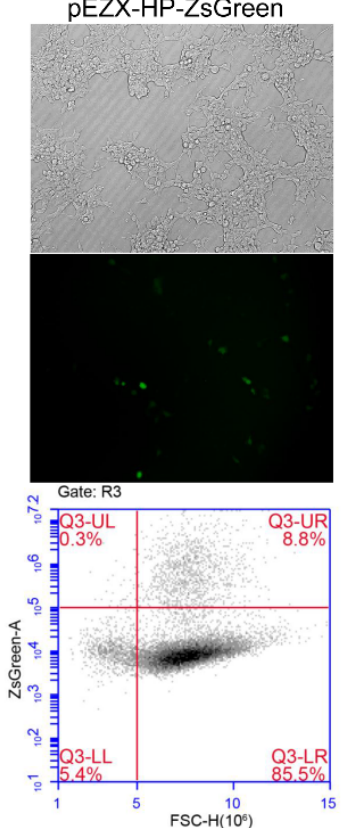
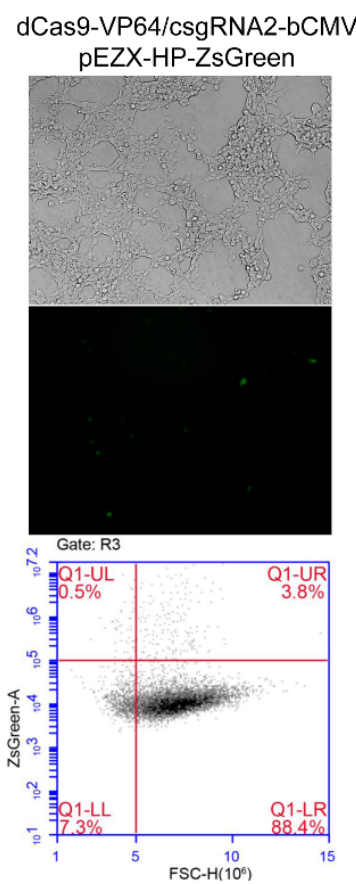

dCas9/csgRNA2-bCMV pEZX-HP-ZsGreen
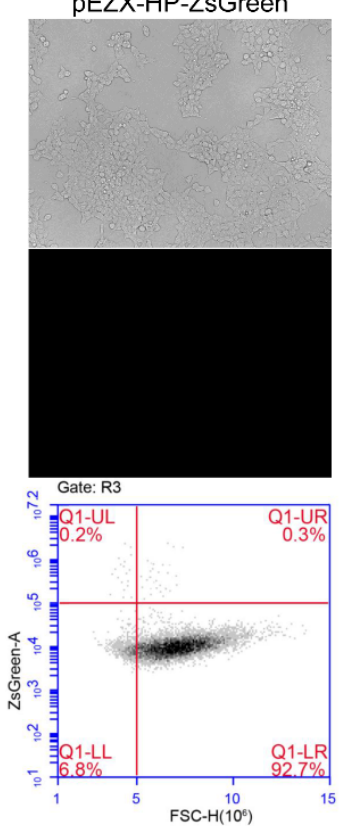

Figure 2. Activation of an exogenous reporter gene ZsGreen under the control of a $H N F 4 \alpha$ promoter by the CRISPR-assistant trans enhancer in 293T cells. Cells were transfected by various vectors. Cells were photographed with a fluorescent microscope and their florescence was analyzed by flow cytometry. In order to reveal the gene activation activity, variant transfections as controls were simultaneously performed. The reporter gene activation efficiency was indicated by the percentage of cells with green fluorescence over the threshold (cells in Q1-UR quadrant).

The transfection of 293T cells revealed that the dCas9-VP64 \& csgRNA2 \& sCMV system has the highest transcriptional activation capability. To verify the versatility of the trans enhancer system in variant cells, we transfected 6 cell lines, including HepG2 (Figure 3), A549 (Supplementary Figure 2), HeLa 
HT29 (Supplementary Figure 6), with the dCas9-VP64 \& csgRNA2 \& sCMV system and reporter construct. As controls, all cells were also co-transfected by dCas9-VP64 \& sgRNA and dCas9 \& csgRNA2 \& SCMV in combination with reporter construct. The results were similar to those of 293T cells. The dCas9VP64 \& csgRNA2 \& sCMV showed the highest transcriptional activation efficiency in all cell lines (Figure 3, Supplementary Figures 2-6). It should be noted that the dCas9 \& csgRNA2 \& sCMV system always showed higher transcriptional activation efficiency than the dCas9-VP64 \& sgRNA in all cell lines (Figure 3, Supplementary Figures 2-6), which confirms the transcriptional activation capability of trans enhancer because the dCas9 \& csgRNA2 \& sCMV system contains no transactivation domain such as VP64. The highest performance of dCas9-VP64 \& csgRNA2 \& sCMV system in all transfected cell lines demonstrates that the trans SCMV has a synergistic effect with the dCas9-fused transactivation domain VP64 in activating gene transcription. These observations were further supported by the quantified fluorescence intensity (MFI) of cells of two biological replicate transfections (Figure 4a).
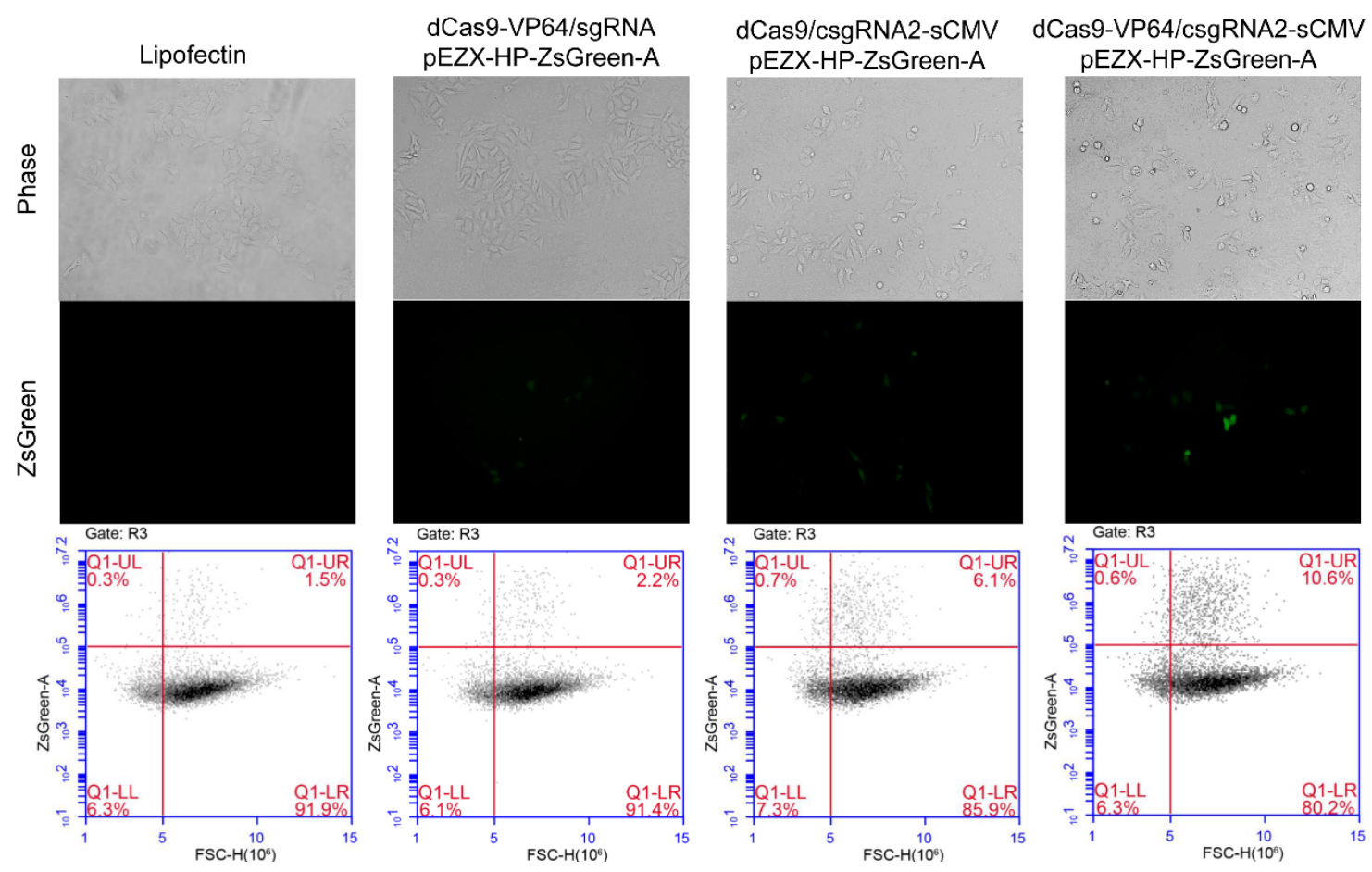

Figure 3. Activation of an exogenous reporter gene ZsGreen under the control of a $H N F 4 \alpha$ promoter by the CRISPR-assistant trans enhancer in the HepG2 cells. Cells were transfected with three transcriptional activation systems, including dCas9-VP64 \& sgRNA, dCas9 \& csgRNA \& sCMV, and dCas9-VP64 \& csgRNA2 \& sCMV, together with a reporter construct (pEZX-HP-ZsGreen-A), respectively. Cells were photographed with a fluorescent microscope and their florescence intensity was analyzed by flow cytometry. The reporter gene activation efficiency was indicated by the percentage of cells with green fluorescence over the threshold (cells in Q1-UR quadrant). 
a
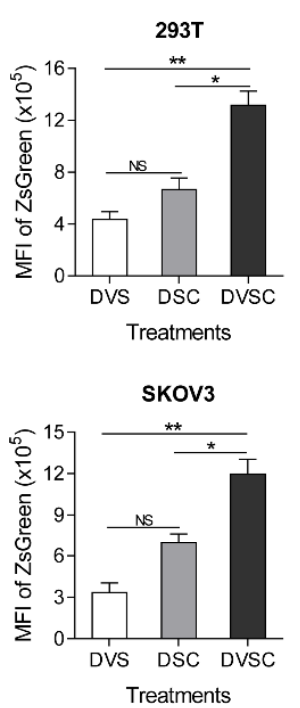

b

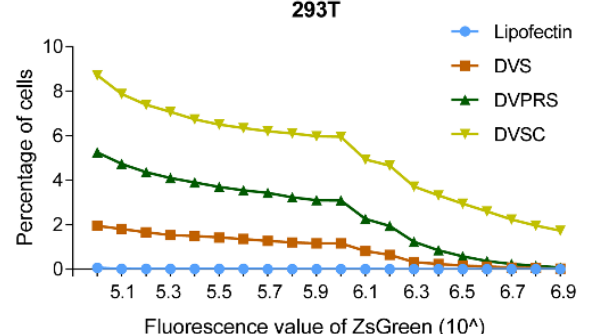

HepG2

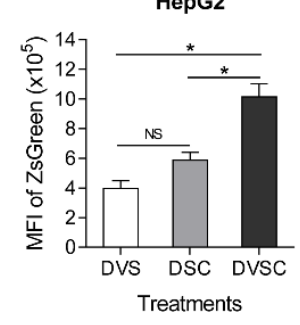

PANC-1

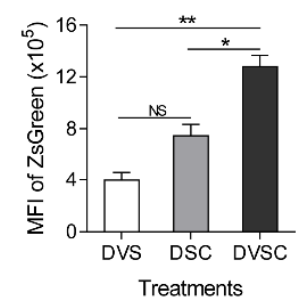

293T

Fluorescence value of ZsGreen $\left(10^{\wedge}\right)$
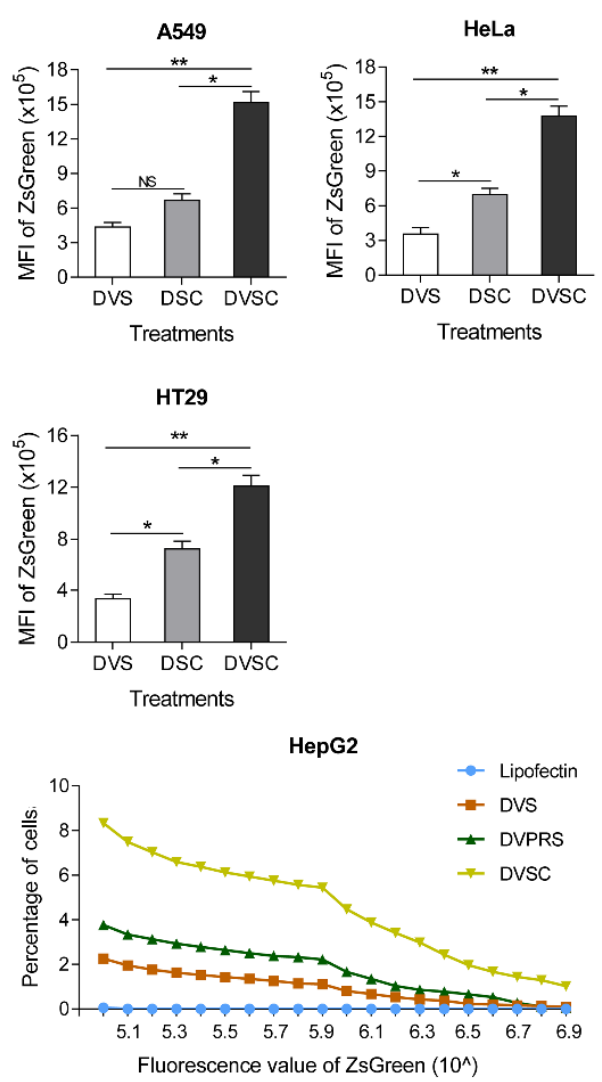

Figure 4. Activation of an exogenous reporter gene ZsGreen under the control of a $H N F 4 \alpha$ promoter by the CRISPR-assistant trans enhancer in multiple cells. a. Transcriptional activation of reporter gene ZsGreen in various cells transfected by different vectors. The florescence intensity of cells were analyzed by flow cytometry and showed as the mean fluorescence intensity (MFI). Co-transfections: DVS, dCas-VP64 \& sgRNA; DSC, dCas9 \& csgRNA \& sCMV; DVSC, dCas-VP64 \& sgRNA \& sCMV. b. Comparison of trans enhancer with VPR. Cells were transfected with three different transcriptional activation systems to activate reporter gene ZsGreen. The florescence intensity of cells was analyzed by flow cytometry and the percentage of cells with particular fluorescence intensity was counted. Transfections: Lipo, lipofection; DVS, dCasVP64 \& sgRNA; DVPRS; dCas9-VPR \& csgRNA; DVSC, dCas9-VP64 \& csgRNA \& sCMV.

\section{Comparison of trans CMV enhancer with VPR}

217 The VPR transcription-activating domain has been shown with higher transcriptional activation ability than

218 VP64. Therefore, we next compared the trans enhancer with this strong transcription activation domain. We transfected the 293T and HepG2 cells with dCas9-VP64/csgRNA, dCas9-VPR/csgRNA, and dCas9VP64/csgRNA \& sCMV, in combination with ZsGreen reporter construct, respectively. The results reveal that dCas9-VPR/csgRNA had better transcriptional activation efficiency than dCas9-VP64/csgRNA as reported by many previous studies (Figure 4b). However, the dCas9-VP64/csgRNA \& sCMV showed far higher transcriptional activation efficiency than dCas9-VPR/csgRNA (Figure 4b). Moreover, we found that the former activated the ZsGreen expression in more cells than the latter at any fluorescence intensity threshold. This means that the trans enhancer sCMV not only make more cells produce fluorescence, but also make more cells produce higher fluorescence than dCas9-VPR/sgRNA. These data further demonstrate the great transcriptional activation ability of CRISPR-assistant trans enhancer. 
229 To investigate the transcriptional activation of endogenous genes by trans enhancer, we chose 10 genes as

230 activating targets, including $H N F 4 \alpha, E 47$ (TCF3), ASCL1, Ngn2, Oct4, Nanog, TNFAIP3, CASP9, CSF3, 231 and Sox2. We designed one promoter-targeting sgRNA for each of these genes. We then transfected 7 232 different cell lines with the dCas9-VP64/csgRNA2 \& sCMV. At the same time, all cells were simultaneously 233 transfected with dCas9-VP64/sgRNA and dCas9/csgRNA \& sCMV as controls. After the gene transcription 234 was detected by qPCR, the expression fold of target genes in the cells transfected by dCas9/csgRNA2 \& 235 sCMV relative to those only transfected by Lipofectamine 2000 was calculated. By comparing the 236 transcriptional activation effects of these 10 genes in different cell lines, we found that the dCas9237 VP64/csgRNA2 \& sCMV always activated the highest transcription of all genes in all cells (Figure 5). As 238 in exogenous gene activation, the dCas9/csgRNA \& sCMV could better activate target genes than dCas9$239 \mathrm{VP} 64 / \mathrm{sgRNA}$ in all cells. Interestingly, we found that the expression of 6 genes including $H N F 4 \alpha, T C F 3$, $240 A S C L 1, N g n 2, T N F A I P 3$, and CSF3 were always highly activated by three systems in all cells. However, the 241 expression of other 4 genes including Oct4, Sox2, Nanog, and CASP9, was just less activated. These results 242 demonstrate that the CRISPR-assistant trans enhancer can activate the expressions of variant endogenous 243 genes in various cells. These results also indicate that the trans SCMV has a synergistic effect with the dCas9244 fused transactivation domain VP64 in activating gene transcription. 
a
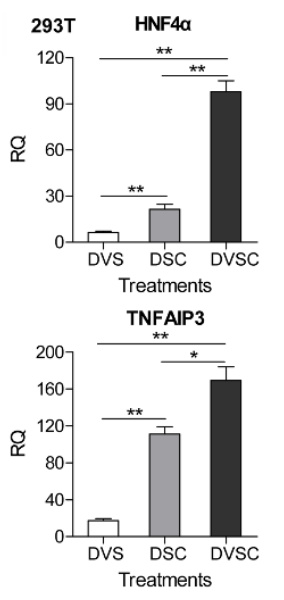

b A549 HNF4a

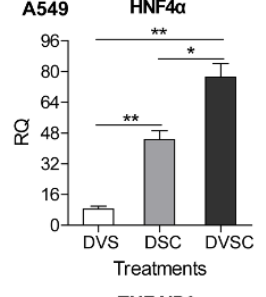

TNFAIP3

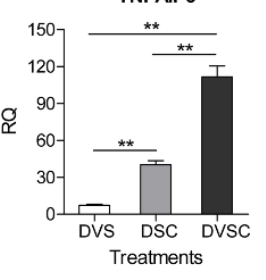

C HeLa HNF4a

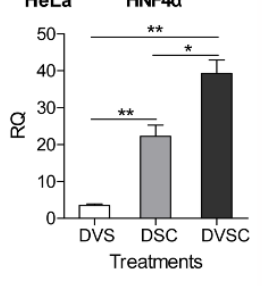

TNFAIP3

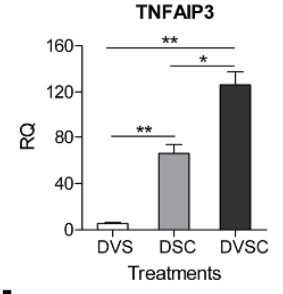

d HepG2 HNF4a

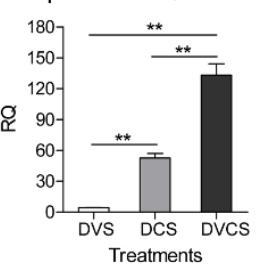

TNFAIP3

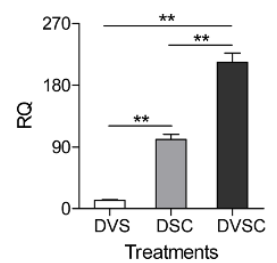

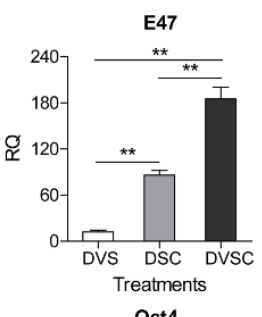
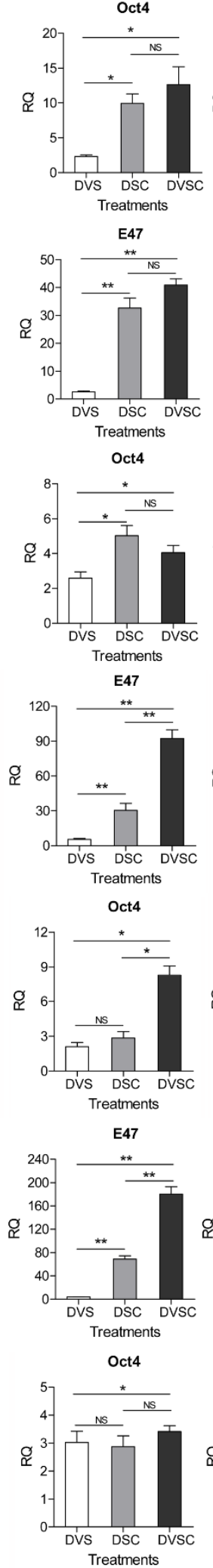
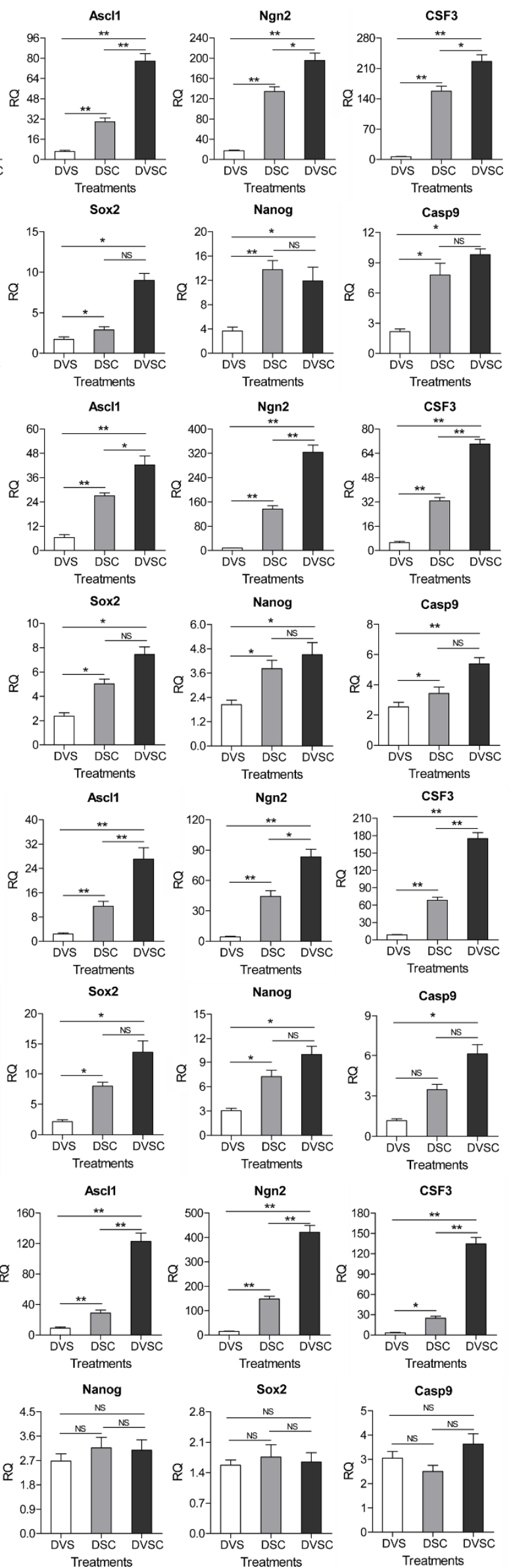
Figure 5. Transcriptional activation of endogenous genes by the CRISPR-assistant trans enhancer. Seven different cell lines were transfected with three different transcriptional activation systems to activate the expression of 10 endogenous genes. The gene transcription was detected by qPCR and the expression level was showed as the relative RNA expression fold to house-keeping gene $\beta$ actin. Three biological replicates were performed for the transfection of each cell line. Data were shown as mean $\pm \mathrm{SD}, \mathrm{n}=3$. The statistical difference was analyzed by Student's t-test. *, $P<0.05$; **, $P<0.01$; NS, no significant statistical difference. Co-transfection: DVS, dCas9-VP64 \& sgRNA; DSC, dCas9 \& csgRNA2 \& sCMV; DVSC, dCas9-VP64 \& csgRNA2 \& sCMV.
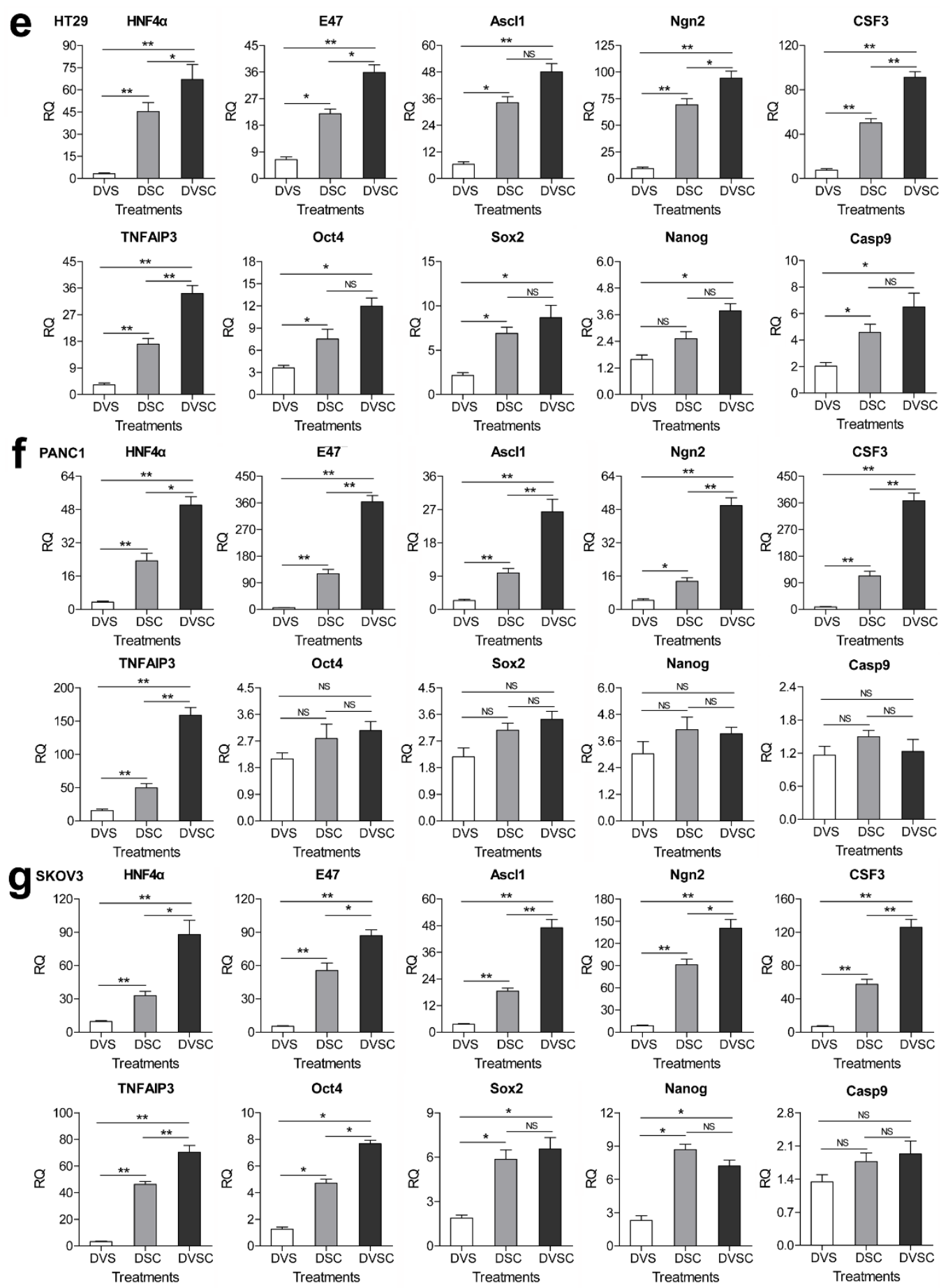

Figure 5-continue. 
Activation of tumor cell differentiation genes by trans enhancer

Finally, we explored whether the trans enhancer can be used to activate critical genes related to tumor differentiation therapy. We selected two transcription factors that were reported to induce tumor cell differentiation, $H N F 4 \alpha$ and $E 47$. The former has been reported to induce the differentiation of cancer cell HepG2, and the latter has been reported to induce the differentiation of cancer cell PANC-1.

We firstly transfected the HepG2 and PANC-1 cells with the dCas9/csgRNA \& sCMV system that targets to a single site in the promoter region of $H N F 4 \alpha$ and $E 47$ genes, respectively. The qPCR detection of gene expression indicates that the two genes were highly activated in the transfected cancer cells (Figure 5; see HepG2 and PANC-1 cells). Moreover, with the activation of HNF4 $\alpha$ in HepG2 cells, the expression of $C D 133$ and $C D 90$ was down-regulated (Figure 6a). In contrast, the expression of $p 21$ was highly upregulated. The expression of some typical genes that are involved in the establishment or maintenance of pluripotency, including Oct3/4, Sox2, Nanog, c-Myc, LIN28, and Klf4, was also down-regulated (Figure 6a). On the contrary, the expression of multiple genes related to healthy liver function, including $G S, B R, A L D O B$, CYP1a2, PEPCK, APOCIII, G-6-P, and HPD, was highly up-regulated (Figure 6b).

Similarly, when the E47 gene was activated in the PANC-1 cell, the expression of $C D 133$ and $C D 90$ was down-regulated (Figure 6c). In contrast, the expression of $p 21$ and TP53INP 1 was highly up-regulated. The expression of some typical genes that are involved in the establishment or maintenance of pluripotency, including Oct3/4, Sox2, Nanog, c-Myc, LIN28, and Klf4, was down-regulated (Figure 6c), while the expression of several genes related to healthy pancreas function, including MIST1, PRSS2, CELA3A, and $C P A 2$, was significantly up-regulated (Figure 6d). The $p 21$ and TP53INP1 was required by E47-induced cell cycle arrest (Figure 6d). The MIST1 normally regulated the acinar maturation pathways. The gene PRSS2, CELA3A, and CPA2 code the digestive enzyme trypsin, elastase 3, and carboxypeptidase A2, respectively.

Besides detecting gene expression, we also detected the physiological phenotypes of transfected cells, including proliferation, migration, and invasion. We characterized the cell proliferation by detecting cell cycles. The results indicate that the $H N F 4 \alpha$ and $E 47$ activation by trans enhancer induced significant cell growth arrest (increased $\mathrm{G}_{0} / \mathrm{G}_{1}$ cells) in HepG2 and PANC-1 cells, respectively (Figure 7a). The woundhealing assay reveals that the migration ability of two cell lines was significantly decreased by the transfection of trans enhancer (Figure 7b, Supplementary Figure 7). The transwell assay also revealed that the trans enhancer transfection resulted in significant decrease of invasion ability of two cell lines 
a
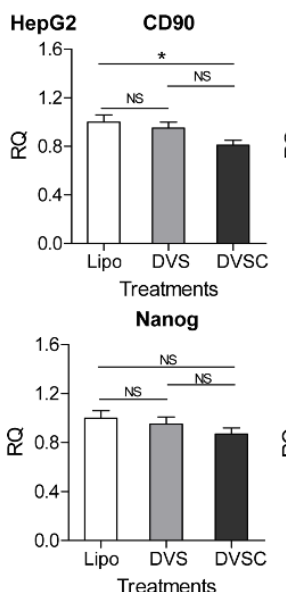

b

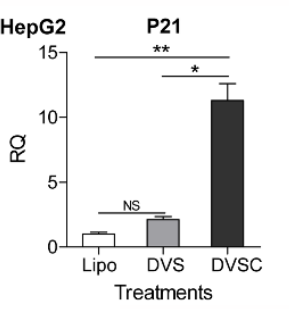

PEPCK

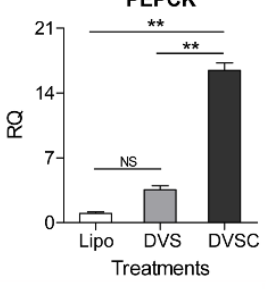

C PANC-1 $\quad$ CD90
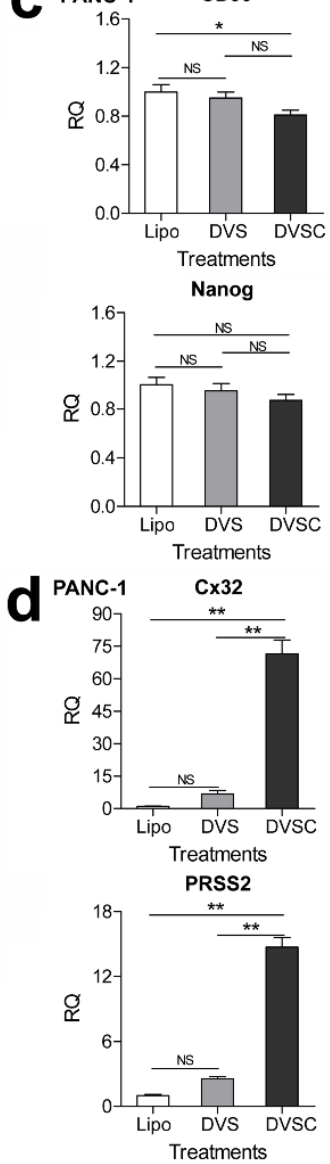
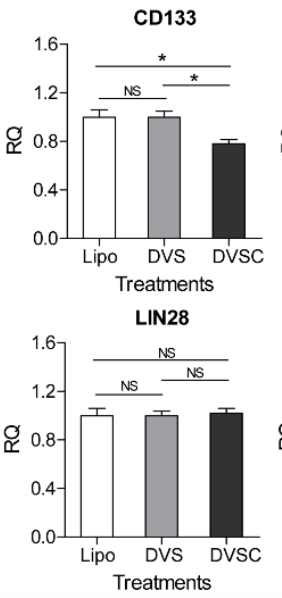

GS

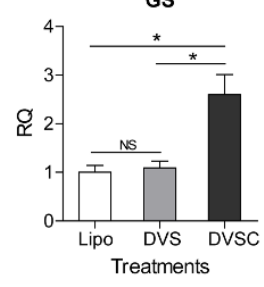

APOCIII

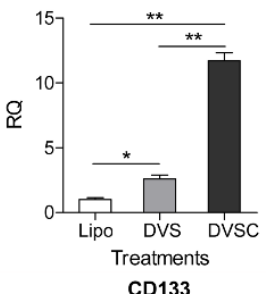

CD133
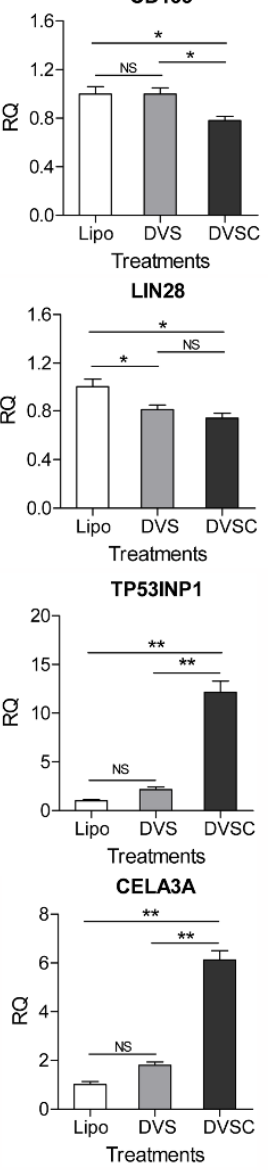
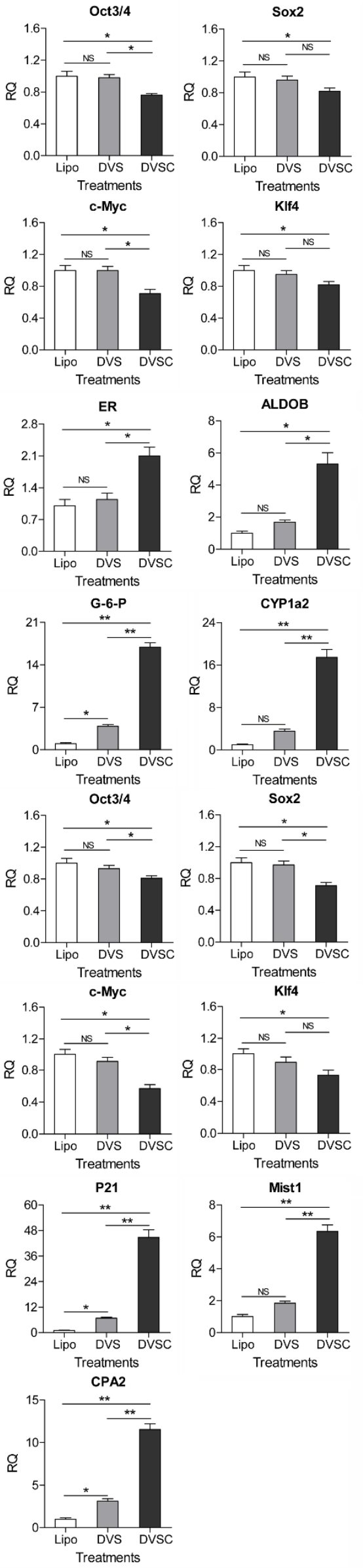
Figure 6. Changes of gene expression and physiological phenotypes in the $H N F 4 \alpha$-activated HepG2 cells and E47-activated PANC-1 cells. a and b. Change of gene expression in the $H N F 4 \alpha$-activated HepG2 cells. $\mathrm{c}$ and $\mathrm{d}$. Change of gene expression in the $E 47$-activated PANC-1 cells. The gene transcription was detected by qPCR and the expression level was showed as the relative RNA expression fold to house-keeping gene $\beta$ actin. Three biological replicates were performed for the transfection of each cell line. Data were shown as mean $\pm \mathrm{SD}, \mathrm{n}=3$. The statistical difference was analyzed by Student's t-test. *, $P<0.05$; **, $P<0.01$; NS, no significant statistical difference. Transfection: Lipo, lipofectin; DVS, dCas9-VP64 \& sgRNA; DVSC, dCas9-VP64 \& csgRNA2 \& sCMV.
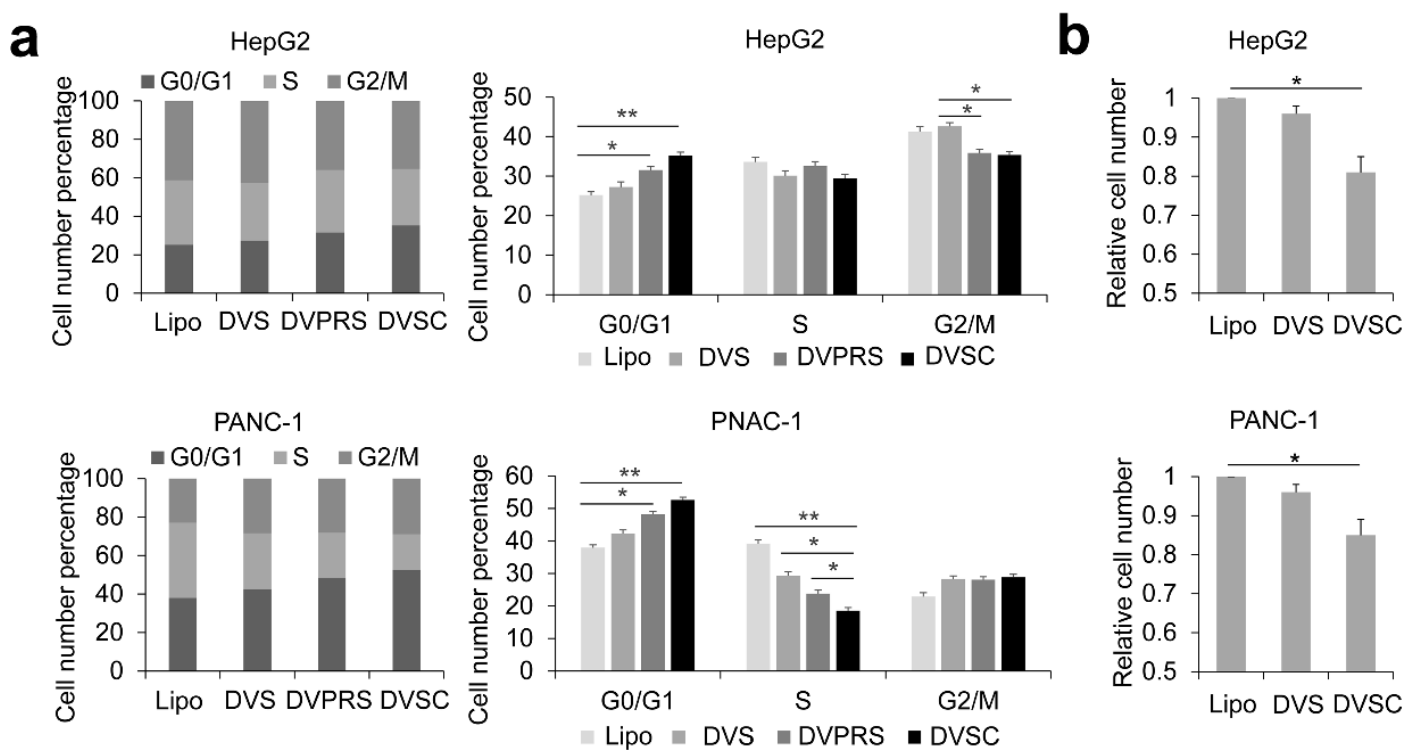

Figure 7. Change of cell cycle and migration in the $H N F 4 \alpha$-activated HepG2 cells and $E 47$-activated PANC1 cells. a. Change of cell cycle in the $H N F 4 \alpha$-activated HepG2 cells and E47-activated PANC-1 cells. b. Change of cell migration in the HNF4 $\alpha$-activated HepG2 cells and E47-activated PANC-1 cells. Three biological replicates were performed for the transfection of each cell line. Data were shown as mean $\pm \mathrm{SD}$, $\mathrm{n}=3$. The statistical difference was analyzed by Student's t-test. ${ }^{*}, P<0.05 ; * *, P<0.01$; NS, no significant statistical difference. Transfection: Lipo, lipofectin; DVS, dCas9-VP64 \& sgRNA; DVPRS, dCas9-VPR \& csgRNA2; DVSC, dCas9-VP64 \& csgRNA2 \& CMV.

\section{Activation of endogenous gene by trans enhancer consisting of VPR and CMV}

Finally, we expected to known whether the combination of dCas9-VPR/csgRNA and sCMV could obtain higher transcriptional activation than the combination of dCas9-VP64/csgRNA and sCMV. We thus transfected 293T cell with dCas9-VP64 and dCas9-VPR together with csgRNA2 and sCMV to activate the endogenous gene $H N F 4 \alpha$. The results indicated that dCas9-VPR/csgRNA \& sCMV always obtained significant lower activation of $H N F 4 \alpha$ expression than dCas9-VP64/csgRNA \& sCMV (Supplementary

Figure 9).

\section{Discussion}

As a typical application, the dCas9-based transcriptional activators have recently been used to reprogram cells in vitro and in vivo for biomedical applications by activating endogenous genes. For example, the fibroblasts can be reprogramed to pluripotency (iPS cell) by activating endogenous Oct4 and Sox2 with 
neuronal cells by activating endogenous Brn2, Ascll, and Myt1l genes with ${ }^{\mathrm{VP} 64} \mathrm{dCas} 9^{\mathrm{VP} 64}$ (Black et al. 2016). The in vivo target genes can be activated by MPH to ameliorate disease phenotypes in mouse models of type I diabetes, acute kidney injury, and muscular dystrophy (Liao et al., 2017). The brain astrocytes can be directly and efficiently converted into functional neurons in vivo by activating Ascl1, Neurog2 and Neurod1 genes with SPH (Zhou et al., 2018). These studies makes CRISPR therapies the grade not the cut (Burgess 2018). However, dCas9-based transcriptional activators are still inefficient at endogenous gene activation and reprogramming (Gao et al., 2014a).

In this study, we developed a new dCas9-based gene activation system by recruiting a CMV enhancer in trans to target gene via dCas9 associated with a simple chimeric sgRNA. This study revealed that the trans enhancer consisted of dCas9-VP64/csgRNA and sCMV could highly activate the expression of variant exogenous and endogenous genes in various mammalian cells, more efficiently than the currently widely used dCas9-VP64 and dCas9-VPR. This study thus developed a new strategy to activating endogenous genes, trans enhancer. To our knowledge, this is the first report to activate gene expression with an enhancer DNA in trans form. This creative strategy has its advantages over the current dCas9-based gene activation systems.

In this study, only one csgRNA was always used to activate all target genes in various cells. This differs from the reported dCas $9 / \mathrm{sgRNA}$ gene activation systems. Currently, multiple sgRNAs have been employed in almost all reported gene activations with various dCas9/sgRNA systems. In general, three or more sgRNAs targeting to an interested gene are often used (Cheng et al., 2013; Maeder et al., 2013; Mali et al. 2013a; Perez-Pinera et al., 2013). In many comparative assays with various numbers of sgRNAs, one sgRNA produced very low or undetectable expression. In comparison with the current multiple sgRNAs strategy, our approach greatly simplified the sgRNA selection, design, and preparation, after all, not all genes are appropriate to design multiple sgRNAs in their promoter region. In addition, expression of multiple sgRNAs are difficult because each sgRNA has to independently transcribed by a long U6 promoter.

It can be seen that our csgRNA is a very simple engineered sgRNA in comparison with the currently reported engineered sgRNAs. The current dCas9/sgRNA activator systems often used a long complexed chimeric sgRNAs that harbor multiple tandem aptamers of various RNA-binding proteins, such as SAM sgRNA (MS2)(Konermann et al., 2015; Liao et al., 2017), Casilio sgRNA (Pumilio/FBF) (Cheng et al., 2016), and scaffold RNAs (MCP, PCP, and Com) (Zalatan et al., 2015). Our csgRNA only harbors a 24-bp short sequence at the end of normal sgRNA. It should be noted that we originally designed three different csgRNAs that have different capture sequences. We found that all of them work in the trans enhancer system; however, one ( $\operatorname{csgRNA2})$ showed the optimal performance. It should be pointed out that the capture sequences were artificially designed so that they have no complementary sequences in human cells, which is important for their high-efficiency specific annealing with sCMV. This study also reveals that the sCMV can anneal with csgRNA in the nuclear of human cells. This interaction successfully recruits the trans enhancer DNA to the target genes by dCas9/csgRNA. Clearly this interaction is more simple than that between RNA aptamers and RNA-binding proteins used in the current sCas9 activation systems. This develops a new approach for biomolecule recruitment in cells, which may be useful for future application.

It is clear that the trans enhancer technique is helpful for in vitro application, such as in vitro cell reprogramming and gene activation for gain-of function. However, it has to say that the trans enhancer still faces difficulty in in vivo application. The trans enhancer used a linear CMV enhancer DNA fragment that has a single-stranded overhang complementary to the $3^{\prime}$ end of csgRNA. It is difficult to produce this kind of trans enhancer DNA in the in vivo cells unless transfecting the in vitro pre-prepared trans enhancer with nanoparticle gene carriers together with expression vectors of dCas9-VP64 and csgRNA. However, the 
vector, virus, such as AAV, that has been approved by FDA to clinical application. Other new strategies should be conceived to address the problem for the in vivo application of trans enhancer. For example, fusing a DNA-binding domain to dCas9 that can bind trans enhancer with its binding sites.

In this study, we selected 10 endogenous genes to be activated by trans enhancer, including $H N F 4 \alpha, E 47$, Ascl1, Ngn2, Oct4, Sox2, Nanog, TNFAIP3(A20), CASP9, and CSF3. These genes are not randomly selected. Most of these genes are transcription factors, including HNF4a, E47, Ascl1, Ngn2, Sox2, Oct4, and Nanog. The combination of Ascl1, Ngn2, and Sox2 has been reported to be used to directly reprogram fibroblasts into nerve cells (Zhao et al. 2015). The combination of Oct4, Sox2, and Nanog has been widely used to reprogram fibroblasts into iPS cells (Takahashi et al. 2007; Takahashi and Yamanaka 2016; Yu et al. 2007). The $H N F 4 \alpha$ and $E 47$ have been used to differentiate the liver and panaceas cancers into normal cells (Kim et al. 2015; Yin et al. 2008). TNFAIP3(A20) is a well-known natural NF-kB inhibitor (Cooper et al. 1996), having the potential to treat NF-kB-over activated diseases such as inflammation and cancers. Caspase 9 is a key gene making cell apoptosis ( $\mathbf{L i}$ et al. 2017). CSF3 codes granulocyte-colony stimulating factor (G-CSF), a glycoprotein that stimulates the bone marrow to produce granulocytes and stem cells and release them into bloodstream (Cetean et al. 2015), which is widely used in chemotherapy to enhancer the immunity of cancer patients. This study revealed that the genes including HNF4a, E47, Ascll, Ngn2, TNFAIP3(A20), and CSF3 were highly activated by trans enhancer in all transfected cells. However, the pluripotency-related genes, including Oct4, Sox2 and Nanog, and CASP 9 were not highly activated as others, which may be related to the tight control of these critical genes.

\section{Materials and methods}

381

382

383

384

385

386

387

388

389

390

391

392

393

394

395

396

397

398

399

400

401

402

403

\section{Vector construction}

To apply the CRISPR/dCas9 expression system to transfection in cells, plasmid containing sgRNA derived by U6 promoter was constructed. We cloned a lac operon sequence with BbsI and BsaI sites at both ends from empty pEASY-Blunt-simple (Transgen, CB101-01) using Pfu High fidelity polymerase (Transgen, AS221-01) with primers Lac-px-F and Lac-px-R (Supplementary Table 1). This lac operon sequence was ligated into px458 (Addgene plasmid ID: 42230) to construct px458-lac. The ligation product was transformed into DH5a and then screened the blue colonies. The px458-lac was verified by sequencing. Then we designed three flanking sequences. The flanking sequence was add to 3 '-end of gRNA scaffold sequence by PCR amplification using a forward primer (U6-F) and one of three reverse primers containing different flanking sequences, U6-1-R, U6-2-R, and U6-3-R (Supplementary Table 1), in which the gRNA scaffold sequence cloned in the px458-lac was used as template. The products were cloned into the pEASY-Bluntsimple, which produced the csgRNA expression vector named as pEASY-csgRNA. The csgRNA refers to capture sgRNA that has a $3^{\prime}$ extended flanking sequence. All flanking sequences were 24-bp in length.

Ten vital genes, including HNF4 , TCF3(E47), ASCL1, Ngn2, Oct4, Nanog, TNFAIP3, CASP9, CSF3, and Sox2, were chosen as target. The sgRNA target sites specific to each gene was designed by CHOPCHOP (https://chopchop.rc.fas.harvard.edu/) . The pEASY-csgRNA expressing a particular csgRNA was constructed with a procedure described by Supplementary Figure 10. The chemically synthesized complementary oligonucleotides (Supplementary Table 2) with a 20-bp target-specific region flanked by two BbsI sites were diluted to $10 \mu \mathrm{M}$ and mixed at the same molar. The final concentration of each oligonucleotide in a reaction was $1 \mu \mathrm{M}$. After mixing well, the complementary oligonucleotides were denatured with high temperature $\left(95^{\circ} \mathrm{C}\right)$ and annealed through a natural cooling process. The hybridization product were diluted 800 times and then ligated into pEASY-csgRNA. The ligation reaction $(10 \mu \mathrm{L})$ consisted of $1 \mu \mathrm{L}$ BbsI, $0.3 \mu \mathrm{L}$ T4 DNA ligase, $1 \mu \mathrm{L} 10 \times$ T4 DNA ligase buffer, $10 \times$ Bovine Serum Albumen 
(final concentration of $0.1 \mathrm{mg} / \mathrm{mL}$ ), $50 \mathrm{ng}$ plasmid pEASY-csgRNA, and $\mathrm{ddH}_{2} \mathrm{O}$ up to $10 \mu 1$ total reaction volume. The ligation reactions was run cycle as fellow: 10 rounds of $37^{\circ} \mathrm{C} 5 \mathrm{~min}$ and $16^{\circ} \mathrm{C} 10 \mathrm{~min}, 37^{\circ} \mathrm{C}$ $30 \mathrm{~min}$, and $80^{\circ} \mathrm{C} 5 \mathrm{~min}$. The ligation product was introduced into DH5 $\alpha$ competent cells and white clones were screened by blue-white screening on LB agar plates with $100 \mu \mathrm{g} / \mathrm{mL}$ Ampicillin, $40 \mu \mathrm{L}$ of $20 \mathrm{mg} / \mathrm{mL}$ $\mathrm{X}$-gal and $40 \mu \mathrm{L}$ of $0.1 \mathrm{M}$ IPTG. The vectors were validated by sequencing. Then the linear sgRNA or csgRNA expression sequences were amplified from the plasmid pEASY-csgRNAs by PCR using a forward primer U6-F and one of reverse primer U6-R, U6-1-R, U6-2-R, and U6-3-R. The primer U6-F and U6-R were used to amplify the normal sgRNA expression template (named as U6-sgRNA), and the primer U6-F and U6-1/2/3-R were used to amplify the csgRNA expression template (named as U6-csgRNA). The PCR products were purified by PCR clean kit (Axygen). These sequences were used to transfect cells as sgRNA transcription templates.

The complete sequence of CMV enhancer/promoter was amplified from pEGFP-N1 by using a forward primer (CMV-F; Supplementary Table 1) and one of reverse primers that had a special flanking sequence (CMV-1-R, CMV-2-R, or CMV-3-R; Supplementary Table 1). The amplified CMV promoter fragment harbors an Nt.BbvCI site at its one end. Then the amplified CMV promoter fragment was digested by Nt.BbvCI. The digested CMV promoter fragment was mixed with a complementary oligonucleotide (CS-1R, CS-2-R, or CS-3-R; Supplementary Table 1) and then denatured at $85^{\circ} \mathrm{C}$ for $10 \mathrm{~min}$. The denatured mixture was then naturally cooled to room temperature. The complementary oligonucleotide has the same sequence with the final $3^{\prime}$ single-strand stick end of CMV promoter fragment. The complementary oligonucleotide was used to remove the denatured oligo from CMV promoter fragment. The CMV promoter fragment was purified with PCR clean kit and used as linear stick-end CMV (sCMV) promoter fragment. The untreated CMV promoter fragment was used as a blunt-end CMV (bCMV) control.

A ZsGreen reporter construct was constructed by cloning an $H N F 4 \alpha$ promoter sequence into the upstream of ZsGreen gene. A 1000-bp HNF4 $\alpha$ promoter sequence was amplified from the genomic DNA of HepG2 cells by PCR using primers HNF4 $\alpha-P-F$ and HNF4 $\alpha-P-R$ (Supplementary Table 1). Then the promoter sequence was ligated into the $\mathrm{pEZX-ZsGreen,} \mathrm{which} \mathrm{produced} \mathrm{an} H N F 4 \alpha$ promoter reporter construct named as pEZX-HP-ZsGreen. The vector pcDNA-dCas9-VP64 was purchased from Addgene (plasmid ID: 47107). The VP64 sequence in the pcDNA-dCas9-VP64 was deleted to construct pcDNA-dCas9. The VP64-p65Rta (VPR) transcription-activating domain sequence was ligated into pcDNA-dCas9 to construct pcDNAdCas9-VPR.

\section{DNA cutting with Cas9/csgRNA}

A sgRNA targeting to the HNF4 $\alpha$ promoter sequence was selected. The sgRNAs were prepared by an in vitro transcription using T7 RNA polymerase (M0251S, NEB). The sgRNA transcription template was prepared by PCR amplification of sgRNA-coding sequence cloned in the sgRNA expression plasmid (pEASY-csgRNA) with a forward primer, HNF4 $\alpha$-T7-F (Supplementary Table 1), which containing a T7 promoter sequence (TAATACGACTCACTATAG, transcription beginning with the $3^{\prime} \mathrm{G}$ ), and one of four reverse primers, U6-R, U6-1-R, U6-2-R, and U6-3-R (Supplementary Table 1). A normal sgRNA (HNF4 $\alpha$ sgRNA) and three csgRNAs (HNF4 $\alpha$-csgRNAs) were prepared. A 732-bp HNF4 $\alpha$ promoter fragments was amplified from pEZX-HP-ZsGreen-A by PCR using primers HNF4 $\alpha$-sP-F and HNF4 $\alpha$-sP-R (Supplementary Table 1). The Cas9 digestion reaction $(30 \mu \mathrm{L})$ consisted of $1 \times$ Cas 9 Nuclease Reaction Buffer, $1 \mu \mathrm{M}$ Cas9 Nuclease (NEB, M0386T), and 300 nM HNF4 $\alpha$-sgRNA or HNF4 $\alpha$-csgRNA. The Cas9 nuclease reaction was firstly incubated at $25{ }^{\circ} \mathrm{C}$ for $10 \mathrm{~min}$. The Cas 9 reaction was then added with $400 \mathrm{ng}$ of purified 732 -bp HNF $4 \alpha$ promoter fragment and incubated at $37^{\circ} \mathrm{C}$ for $15 \mathrm{~min}$. Finally, Cas 9 nuclease was inactivated at $65^{\circ} \mathrm{C}$ for $10 \mathrm{~min}$. The reaction was run with $1.5 \%$ agarose gel electrophoresis. 

set at $37{ }^{\circ} \mathrm{C}$ and $5 \% \mathrm{CO}_{2}$. Cells were cultured in the Dulbecco's Modified Eagle Medium (DMEM) or Roswell Park Memorial Institute (RPMI) 1640 medium supplemented with 10\% FBS, 100 units $/ \mathrm{mL}$ penicillin, and $100 \mu \mathrm{g} / \mathrm{mL}$ streptomycin. Cells at $>70 \%$ confluency in each well of 12 -well plate were transfected with $800 \mathrm{ng}$ total DNA, including $500 \mathrm{ng}$ plasmid (pcDNA-dCas9, pcDNA-dCas9-VP64, or pcDNA-dCas9-VPR), 150 ng linear sgRNA expression template (U6-sgRNA or U6-csgRNA), and $150 \mathrm{ng}$ linear CMV, by Lipofectamine ${ }^{\circledR} 2000$ (ThermoFisher Scientific) using the following protocol. For each transfection, cells were cultured with $600 \mu \mathrm{L}$ of Opti-MEM (ThermoFisher Scientific) at $37{ }^{\circ} \mathrm{C}$ for $0.5 \mathrm{~h}$ when cell grew to a density of $4 \times 10^{5} /$ well. A stock solution of $100 \mu \mathrm{L}$ of Opti-MEM, $800 \mathrm{ng}$ of total DNA, $100 \mu \mathrm{L}$ of Opti-MEM, and $4 \mu \mathrm{L}$ of Lipofectamine 2000 was made for per transfection. The solution was then vortexed and incubated for $5 \mathrm{~min}$ at room temperature. Thereafter, the Opti-MEM/Lipofectamine solution was added to the individual aliquots of DNA stocked in $100 \mu \mathrm{L}$ of Opti-MEM, vortexed, and incubated for $20 \mathrm{~min}$ at room temperature before being added to each well. After incubated cell with transfection solution for $4 \mathrm{~h}$, the medium of each well was replaced with $800 \mu \mathrm{L}$ of fresh DMEM or RPMI 1640 medium containing $10 \%$ FBS. The cells were further incubated at $37{ }^{\circ} \mathrm{C}$ and $5 \% \mathrm{CO}_{2}$ for another $36 \mathrm{~h}$. At the end of experiment, all cells were observed and photographed under a fluorescence microscope (Olympus) at $200 \times$ magnification.

CMV linear fragment with sticky end (sCMV) or with blunt end (bCMV) were used as activating factor mixed with other ingredients. In activating the $H N F 4 \alpha$ promoter reporter construct, the 293T cells were cotransfected with the HNF4 $\alpha$-sgRNA, pcDNA-dCas9-VP64, and pEZX-HP-ZsGreen as the VP64 control group, and the 293T cells were co-transfected with the HNF4 $\alpha$-csgRNA, pcDNA-dCas9-VP64, CMV linear fragment, and pEZX-HP-ZsGreen as trans-CMV group. In activating endogenous genes, cells were cotransfected with the sgRNA and pcDNA-dCas9-VP64 as the VP64 control group, and cells were cotransfected with the csgRNA, pcDNA-dCas9-VP64, and CMV linear fragment as trans-CMV group.

The fluorescence intensity of cells was quantified with flow cytometry (Calibur, BD, USA). Ten thousand cells were measured for each transfection. Flow cytometry data analysis and figure preparation were done using BD software.

\section{Quantitative PCR}

479 Total RNA was extracted from transfected cell using TRIzolTM (Invitrogen). cDNA was synthesized from up to $3 \mu \mathrm{g}$ total RNA in a volume of $20 \mu \mathrm{l}$ consisting of $1 \times$ Hifair $^{\text {TM }}$ III SuperMix (YEASEN, 11137ES50). All mRNA levels were normalized using GADPH mRNA as a control. Transcription levels of differently treated cells were analyzed by quantitative PCR using ABI Step One Plus (Applied Biosystems) according to the manufacturer's protocol. The quantitative PCR used the primers listed in Supplementary Table 2 and 3.

\section{Wound healing migration assays}

486 Cells were seeded in 24-well plates (Costar, Cambridge, MA) and grown to confluence (overnight). Then the cells were transfected with dCas9-VP64/sgRNA or dCas9-VP64/csgRNA \& sCMV to activate the target genes. The cells were transfected for 4 hs. Then the transfection medium was removed and cells were cultured with the fresh complete medium for $36 \mathrm{hs}$. Cell monolayers were wounded with a sterile pipette tip 
media for $48 \mathrm{hs}$ and images by microscope.

Cells were transfected with or without trans CMV enhancer to activate the target genes. After transfected 36 hours, the cells were removed medium and washed three times with PBS. Then cells were trypsinized and resuspended with medium. The resuspended cells diluted to $1 \times 10^{5} \mathrm{cells} / \mathrm{mL}$. In $24-$ well plate, each well was added $1 \mathrm{~mL}$ medium, and then transwell chamber was added into the well. Finally, $2 \times 10^{4}$ cells $(200 \mu \mathrm{L})$ were added into each transwell. Then the 24 -well plate was maintained in an incubator set at $37{ }^{\circ} \mathrm{C}$ and $5 \%$ $\mathrm{CO}_{2}$ for 48 hs. The cells invaded through the transwell chamber and adhered to the surface of the well were fixed and stained with acridine orange and imaged with fluorescence microscope. Cells in acridine orangestained images were counted by ImageJ.

\section{Acridine orange staining}

502 After transfection and culturing in fresh medium, each cell line was washed twice with PBS, then stained with $100 \mu \mathrm{g} / \mathrm{mL}$ acridine orange (AO) for $10 \mathrm{~min}$ at room temperature. Then cells were observed and photographed under a fluorescence microscope (Olympus) at $200 \times$ magnification.

\section{Statistical analyses}

506 Each cell transfection for detecting gene expression activation by trans-enhancer was performed in three biological replicates. In each biological replicate, at least three technical replicates were performed. In qPCR detection of gene expression, the mean RQ value of technical replicates was used as the RQ value of one biological replicate. The mean RQ value of three biological replicates were used to calculate the final mean and standard deviation (SD). Data were analyzed by Student's t-test when comparing 2 groups. Data were shown as mean $\pm \mathrm{SD}$ and differences were considered significant at $P<0.05$.

\section{Acknowledgments}

513 This work was supported by the grants from the National Natural Science Foundation of China (Grant 514 61571119).

\section{Author contributions}

516 X.H.X. performed experiments, analyzed data and wrote the manuscript. D.Y.W., W.D. and J.W. assisted 517 with the preparation of reagents, vector construction, data analysis and manuscript preparation. J.K.W. 518 conceptualized the project, designed and supervised the research, and wrote the manuscript. J.K.W. provided 519 financial support for the project.

\section{Competing interests}

521 The authors declare no competing interests.

\section{Additional files}

523 Supplementary files

524 The file contains three tables, ten figures, and vector sequences.

\section{Reference} 2016. Targeted Epigenetic Remodeling of Endogenous Loci by CRISPR/Cas9-Based Transcriptional 
doi:10.1016/j.stem.2016.07.001

Boshart M, Weber F, Jahn G, Dorschhasler K, Fleckenstein B, Schaffner W. 1985. A Very Strong Enhancer Is Located Upstream of an Immediate Early Gene of Human Cytomegalo-Virus. Cell 41:521-530. doi:Doi 10.1016/S0092-8674(85)80025-8

Burgess DJ. 2018. Translational genetics: CRISPR therapies - making the grade not the cut. Nature Reviews Genetics 19:63. doi:10.1038/nrg.2017.112

Carter D, Chakalova L, Osborne CS, Dai YF, Fraser P. 2002. Long-range chromatin regulatory interactions in vivo. Nature Genetics 32:623-626. doi:10.1038/ng1051

Cetean S, Cainap C, Constantin AM, Cainap S, Gherman A, Oprean L, Hangan A, Oprean R. 2015. The importance of the granulocyte-colony stimulating factor in oncology. Clujul Med 88:468-472. doi:10.15386/cjmed-531

Chavez A, Scheiman J, Vora S, Pruitt BW, Tuttle M, E PRI, Lin S, Kiani S, Guzman CD, Wiegand DJ, TerOvanesyan D, Braff JL, Davidsohn N, Housden BE, Perrimon N, Weiss R, Aach J, Collins JJ, Church GM. 2015. Highly efficient Cas9-mediated transcriptional programming. Nature Methods 12:326-328. doi:10.1038/nmeth.3312

Chavez A, Tuttle M, Pruitt BW, Ewen-Campen B, Chari R, Ter-Ovanesyan D, Haque SJ, Cecchi RJ, Kowal EJK, Buchthal J, Housden BE, Perrimon N, Collins JJ, Church G. 2016. Comparison of Cas9 activators in multiple species. Nature Methods 13:563-567. doi:10.1038/nmeth.3871

Chen M, Qi LS. 2017. Repurposing CRISPR System for Transcriptional Activation. Advances in Experimental Medicine and Biology 983:147-157. doi:10.1007/978-981-10-4310-9_10

Cheng AW, Jillette N, Lee P, Plaskon D, Fujiwara Y, Wang W, Taghbalout A, Wang H. 2016. Casilio: a versatile CRISPR-Cas9-Pumilio hybrid for gene regulation and genomic labeling. Cell Research 26:254-257. doi: $10.1038 / \mathrm{cr} .2016 .3$

Cheng AW, Wang H, Yang H, Shi L, Katz Y, Theunissen TW, Rangarajan S, Shivalila CS, Dadon DB, Jaenisch R. 2013. Multiplexed activation of endogenous genes by CRISPR-on, an RNA-guided transcriptional activator system. Cell Research 23:1163-1171. doi:10.1038/cr.2013.122

Choudhury SR, Cui Y, Lubecka K, Stefanska B, Irudayaraj J. 2016. CRISPR-dCas9 mediated TET1 targeting for selective DNA demethylation at BRCA1 promoter. Oncotarget 7:46545-46556. doi:10.18632/oncotarget.10234

Cooper JT, Stroka DM, Brostjan C, Palmetshofer A, Bach FH, Ferran C. 1996. A20 blocks endothelial cell activation through a NF-kappaB-dependent mechanism. Journal of Biological Chemistry 271:18068-18073.

Dominguez AA, Lim WA, Qi LS. 2015. Beyond editing: repurposing CRISPR-Cas9 for precision genome regulation and interrogation. Nature Reviews Molecular Cell Biology 17:5. doi:10.1038/nrm.2015.2

Doudna JA, Charpentier E. 2014. The new frontier of genome engineering with CRISPR-Cas9. Science 346.

Foecking MK, Hofstetter H. 1986. Powerful and versatile enhancer-promoter unit for mammalian expression vectors. Gene 45:101-105.

Furth PA, Hennighausen L, Baker C, Beatty B, Woychick R. 1991. The variability in activity of the universally expressed human cytomegalovirus immediate early gene 1 enhancer/promoter in transgenic mice. Nucleic Acids Res 19:6205-6208.

Gao X, Tsang JC, Gaba F, Wu D, Lu L, Liu P. 2014a. Comparison of TALE designer transcription factors and the CRISPR/dCas9 in regulation of gene expression by targeting enhancers. Nucleic Acids Research 42:e155. doi:10.1093/nar/gku836

Gao X, Tsang JCH, Gaba F, Wu D, Lu L, Liu P. 2014b. Comparison of TALE designer transcription factors and the CRISPR/dCas9 in regulation of gene expression by targeting enhancers. Nucleic Acids Research 42:e155- 
e155. doi:10.1093/nar/gku836

Hilton IB, D'Ippolito AM, Vockley CM, Thakore PI, Crawford GE, Reddy TE, Gersbach CA. 2015. Epigenome editing by a CRISPR-Cas9-based acetyltransferase activates genes from promoters and enhancers. Nature Biotechnology 33:510-517. doi:10.1038/nbt.3199

Ho SCL, Mariati, Yeo JHM, Fang SG, Yang YS. 2015. Impact of Using Different Promoters and Matrix Attachment Regions on Recombinant Protein Expression Level and Stability in Stably Transfected CHO Cells. Mol Biotechnol 57:138-144. doi:DOI 10.1007/s12033-014-9809-2

Jinek M, Chylinski K, Fonfara I, Hauer M, Doudna JA, Charpentier E. 2012. A Programmable Dual-RNA-Guided DNA Endonuclease in Adaptive Bacterial Immunity. Science 337:816.

Kearns NA, Pham H, Tabak B, Genga RM, Silverstein NJ, Garber M, Maehr R. 2015. Functional annotation of native enhancers with a Cas9-histone demethylase fusion. Nature Methods 12:401-403. doi:10.1038/nmeth.3325

Kiani S, Chavez A, Tuttle M, Hall RN, Chari R, Ter-Ovanesyan D, Qian J, Pruitt BW, Beal J, Vora S, Buchthal J, Kowal EJK, Ebrahimkhani MR, Collins JJ, Weiss R, Church G. 2015. Cas9 gRNA engineering for genome editing, activation and repression. Nature Methods 12:1051. doi:10.1038/nmeth.3580

https://www.nature.com/articles/nmeth.3580\#supplementary-information

Kim DW, Uetsuki T, Kaziro Y, Yamaguchi N, Sugano S. 1990. Use of the Human Elongation Factor-1 $\alpha$ Promoter as a Versatile and Efficient Expression System. Gene 91:217-223. doi:Doi 10.1016/0378-1119(90)90091-5

Kim S, Lahmy R, Riha C, Yang C, Jakubison BL, van Niekerk J, Staub C, Wu Y, Gates K, Dong DS, Konieczny SF, Itkin-Ansari P. 2015. The Basic Helix-Loop-Helix Transcription Factor E47 Reprograms Human Pancreatic Cancer Cells to a Quiescent Acinar State With Reduced Tumorigenic Potential. Pancreas 44:718727. doi:10.1097/MPA.0000000000000328

Konermann S, Brigham MD, Trevino AE, Joung J, Abudayyeh OO, Barcena C, Hsu PD, Habib N, Gootenberg JS, Nishimasu H, Nureki O, Zhang F. 2015. Genome-scale transcriptional activation by an engineered CRISPR-Cas9 complex. Nature 517:583-588. doi:10.1038/nature14136

Li P, Zhou L, Zhao T, Liu X, Zhang P, Liu Y, Zheng X, Li Q. 2017. Caspase-9: structure, mechanisms and clinical application. Oncotarget 8:23996-24008. doi:10.18632/oncotarget.15098

Liao HK, Hatanaka F, Araoka T, Reddy P, Wu MZ, Sui Y, Yamauchi T, Sakurai M, O'Keefe DD, Nunez-Delicado E, Guillen P, Campistol JM, Wu CJ, Lu LF, Esteban CR, Izpisua Belmonte JC. 2017. In Vivo Target Gene Activation via CRISPR/Cas9-Mediated Trans-epigenetic Modulation. Cell 171:1495-1507 e1415. doi:10.1016/j.cell.2017.10.025

Liu P, Chen M, Liu Y, Qi LS, Ding S. 2018. CRISPR-Based Chromatin Remodeling of the Endogenous Oct4 or Sox2 Locus Enables Reprogramming to Pluripotency. Cell Stem Cell 22:252-261 e254. doi:10.1016/j.stem.2017.12.001

Liu XS, Wu H, Ji X, Stelzer Y, Wu X, Czauderna S, Shu J, Dadon D, Young RA, Jaenisch R. 2016a. Editing DNA Methylation in the Mammalian Genome. Cell 167:233-247 e217. doi:10.1016/j.cell.2016.08.056

Liu Y, Zhan Y, Chen Z, He A, Li J, Wu H, Liu L, Zhuang C, Lin J, Guo X, Zhang Q, Huang W, Cai Z. 2016 b. Directing cellular information flow via CRISPR signal conductors. Nature Methods 13:938-944. doi:10.1038/nmeth.3994

Lu J, Zhao C, Zhao Y, Zhang J, Zhang Y, Chen L, Han Q, Ying Y, Peng S, Ai R, Wang Y. 2018. Multimode drug inducible CRISPR/Cas9 devices for transcriptional activation and genome editing. Nucleic Acids Research 46:e25. doi:10.1093/nar/gkx1222

Maeder ML, Linder SJ, Cascio VM, Fu Y, Ho QH, Joung JK. 2013. CRISPR RNA-guided activation of endogenous human genes. Nature Methods 10:977-979. doi:10.1038/nmeth.2598 
Mali P, Aach J, Stranges PB, Esvelt KM, Moosburner M, Kosuri S, Yang L, Church GM. 2013a. CAS9 transcriptional activators for target specificity screening and paired nickases for cooperative genome engineering. Nature Biotechnology 31:833-838. doi:10.1038/nbt.2675

Mali P, Esvelt KM, Church GM. 2013b. Cas9 as a versatile tool for engineering biology. Nature Methods 10:957963. doi:10.1038/nmeth.2649

Mali P, Yang L, Esvelt KM, Aach J, Guell M, DiCarlo JE, Norville JE, Church GM. 2013c. RNA-Guided Human Genome Engineering via Cas9. Science 339:823.

McClellan JS, Dove C, Gentles AJ, Ryan CE, Majeti R. 2015. Reprogramming of primary human Philadelphia chromosome-positive B cell acute lymphoblastic leukemia cells into nonleukemic macrophages. Proceedings of the National Academy of Sciences of the United States of America 112:4074-4079. doi:10.1073/pnas.1413383112

Nihongaki Y, Yamamoto S, Kawano F, Suzuki H, Sato M. 2015. CRISPR-Cas9-based photoactivatable transcription system. Chemistry \& Biology 22:169-174. doi:10.1016/j.chembiol.2014.12.011

Perez-Pinera P, Kocak DD, Vockley CM, Adler AF, Kabadi AM, Polstein LR, Thakore PI, Glass KA, Ousterout DG, Leong KW, Guilak F, Crawford GE, Reddy TE, Gersbach CA. 2013. RNA-guided gene activation by CRISPR-Cas9-based transcription factors. Nature Methods 10:973-976. doi:10.1038/nmeth.2600

Perrin A, Rousseau J, Tremblay JP. 2017. Increased Expression of Laminin Subunit Alpha 1 Chain by dCas9VP160. Mol Ther Nucleic Acids 6:68-79. doi:10.1016/j.omtn.2016.11.004

Polstein LR, Gersbach CA. 2015. A light-inducible CRISPR-Cas9 system for control of endogenous gene activation. Nature Chemical Biology 11:198-200. doi:10.1038/nchembio.1753

Radzisheuskaya A, Shlyueva D, Müller I, Helin K. 2016. Optimizing sgRNA position markedly improves the efficiency of CRISPR/dCas9-mediated transcriptional repression. Nucleic Acids Research 44:e141-e141. doi:10.1093/nar/gkw583

Sagiv-Barfi I, Czerwinski DK, Levy S, Alam IS, Mayer AT, Gambhir SS, Levy R. 2018. Eradication of spontaneous malignancy by local immunotherapy. Sci Transl Med 10. doi:10.1126/scitranslmed.aan 4488

Saunderson EA, Stepper P, Gomm JJ, Hoa L, Morgan A, Allen MD, Jones JL, Gribben JG, Jurkowski TP, Ficz G. 2017. Hit-and-run epigenetic editing prevents senescence entry in primary breast cells from healthy donors. Nature Communications 8:1450. doi:10.1038/s41467-017-01078-2

Schmidt EV, Christoph G, Zeller R, Leder P. 1990. The cytomegalovirus enhancer: a pan-active control element in transgenic mice. Mol Cell Biol 10:4406-4411.

Takahashi K, Tanabe K, Ohnuki M, Narita M, Ichisaka T, Tomoda K, Yamanaka S. 2007. Induction of pluripotent stem cells from adult human fibroblasts by defined factors. Cell 131:861-872. doi:10.1016/j.cell.2007.11.019

Takahashi K, Yamanaka S. 2016. A decade of transcription factor-mediated reprogramming to pluripotency. Nature Reviews Molecular Cell Biology 17:183-193. doi:10.1038/nrm.2016.8

Tanenbaum ME, Gilbert LA, Qi LS, Weissman JS, Vale RD. 2014. A protein tagging system for signal amplification in gene expression and fluorescence imaging. Cell 159:635-646. doi:10.1016/j.cell.2014.09.039

Tolhuis B, Palstra RJ, Splinter E, Grosveld F, de Laat W. 2002. Looping and interaction between hypersensitive sites in the active beta-globin locus. Molecular Cell 10:1453-1465.

Wang D, Dai W, Wu J, Wang J. 2018. Improving transcriptional activity of human cytomegalovirus major immediate-early promoter by mutating NF-kappaB binding sites. Protein Expression and Purification 142:16-24. doi:10.1016/j.pep.2017.09.008

Wang H, Russa ML, Qi LS. 2016. CRISPR/Cas9 in Genome Editing and Beyond. Annual Review of Biochemistry 85:227-264. doi:10.1146/annurev-biochem-060815-014607 
Yin C, Lin Y, Zhang X, Chen Y-X, Zeng X, Yue H-Y, Hou J-L, Deng X, Zhang J-P, Han Z-G, Xie W-F. 2008. Differentiation therapy of hepatocellular carcinoma in mice with recombinant adenovirus carrying hepatocyte nuclear factor-4 $\alpha$ gene. Hepatology 48:1528-1539. doi:10.1002/hep.22510

Yu J, Vodyanik MA, Smuga-Otto K, Antosiewicz-Bourget J, Frane JL, Tian S, Nie J, Jonsdottir GA, Ruotti V, Stewart R, Slukvin, II, Thomson JA. 2007. Induced pluripotent stem cell lines derived from human somatic cells. Science 318:1917-1920. doi:10.1126/science.1151526

Zalatan JG, Lee ME, Almeida R, Gilbert LA, Whitehead EH, La Russa M, Tsai JC, Weissman JS, Dueber JE, Qi LS, Lim WA. 2015. Engineering complex synthetic transcriptional programs with CRISPR RNA scaffolds. Cell 160:339-350. doi:10.1016/j.cell.2014.11.052

Zhao P, Zhu T, Lu X, Zhu J, Li L. 2015. Neurogenin 2 enhances the generation of patient-specific induced neuronal cells. Brain Research 1615:51-60. doi:10.1016/j.brainres.2015.04.027

Zheng Y, Shen W, Zhang J, Yang B, Liu YN, Qi H, Yu X, Lu SY, Chen Y, Xu YZ, Li Y, Gage FH, Mi S, Yao J. 2018. CRISPR interference-based specific and efficient gene inactivation in the brain. Nature Neuroscience 21:447-454. doi:10.1038/s41593-018-0077-5 


\section{Supplementary infromation}

Supplementary Tables (Primer used in this study)

Supplementary Table 1. Primers were used in high fidelity amplification in this study.

\begin{tabular}{|c|c|}
\hline Name & Sequence \\
\hline Lac-px-F & 5'-ACGGTCTCGCACCGGGTCTTCAGCGCCCAATACGCAAACCGCCTC-3' \\
\hline Lac-px-R & 5'-TCGGTCTCCAAACAGGTCTTCGCGTCCATTCGCCATTCAGGCTGCGC-3' \\
\hline U6-F & 5'-GAGGGCCTATTTCCCATGATTCC-3' \\
\hline U6-R & 5'-AAAAAAGCACCGACTCGGTGCCACTTTTTC-3' \\
\hline U6-1-R & 5'-AAAAAAAAGGTATGTTTGCCGTCGGCCCGATTGCACCGACTCGGTGCCACTTTTTC-3' \\
\hline U6-2-R & 5'-AAAAAAAAGGTATGTTTGCCGTCGGCCCGATTGCACCGACTCGGTGCCACTTTTTC-3' \\
\hline U6-3-R & 5'-AAAAAAAAAGGTATGTTTGAGGTTCCACTAGAGCACCGACTCGGTGCCACTTTTTC-3' \\
\hline dCas9-X-F & 5'-CCGCTCGAGGCCACCATGGACTACAAAGACCATG-3' \\
\hline dCas9-N-R & 5'-ATAAGAATGCGGCCGCTCAAACTTTGCGTTTCTTTTTCGGGCTAGC-3' \\
\hline HNF4 $\alpha-\mathrm{T} 7-\mathrm{F}$ & 5'-TTCTAATACGACTCACTATAGGGAGCAGGTTGAATTTAGAATGG-3' \\
\hline HNF4 $\alpha-P-F$ & 5'-CCGCTCGAGTGAGATCCAAAACTGAGACAAAAGAAACGGGGCTG-3' \\
\hline HNF4 $\alpha-P-R$ & 5'-CCCAAGCTTAAGCCCACCCAGCCGGAGAGCTGGGGGCATGGAGG-3' \\
\hline $\mathrm{HNF} 4 \alpha-\mathrm{sP}-\mathrm{F}$ & 5'-TAGTAGGTGCTCAATAAATATGTTAG-3' \\
\hline HNF4 $\alpha-\mathrm{sP}-\mathrm{R}$ & 5'-GGCTTTACCAACAGTACCGGATCT-3' \\
\hline CMV-F & 5'-TAGTTATTAATAGTAATCAATTACGGGG-3' \\
\hline CMV-1-R & 5'-AATCGGGCCGACGGCAAACATACCCCTCAGCAGCTCTGCTTATATAGACCTCCCACC-3' \\
\hline CMV-2-R & 5'-GGAACCTTACGAATACCAGATGCTCCTCAGCAGCTCTGCTTATATAGACCTCCCACC-3' \\
\hline CMV-3-R & 5'-ATCTAGTGGAACCTCAAACATACCCCTCAGCAGCTCTGCTTATATAGACCTCCCACC-3' \\
\hline CS-1-R & 5'-GGGGTATGTTTGCCGTCGGCCCGATT-3' \\
\hline CS-2-R & 5'-GGAGCATCTGGTATTCGTAAGGTTCC-3' \\
\hline CS-3-R & 5'-GGGGTATGTTTGAGGTTCCACTAGAT-3' \\
\hline
\end{tabular}


bioRxiv preprint doi: https://doi.org/10.1101/517920; this version posted January 11,2019 . The copyright holder for this preprint (which was not certified by peer review) is the author/funder. All rights reserved. No reuse allowed without permission.

Supplementary Table 2. Oligonucleotide used to prepare target-specific regions (20 bp) of sgRNA

\begin{tabular}{|c|c|c|}
\hline Gene & Name & Sequence \\
\hline \multirow{2}{*}{ HNF4 $\alpha$} & $\mathrm{HNF} 4 \alpha$-sg-S & 5'-AGGAAGACGGCACCGGCCAGTCACTTAGGGAACCCGGTTTGGGTCTTCGA-3' \\
\hline & HNF4 $\alpha$-sg-AS & 5'-TCGAAGACCCAAACCGGGTTCCCTAAGTGACTGGCCGGTGCCGTCTTCCT-3' \\
\hline \multirow{2}{*}{$\begin{array}{l}\text { E47 } \\
\text { ( TCF3) }\end{array}$} & TCF3-sg-S & 5'-AGGAAGACGGCACCGGGCGCACGGGCCCCGCGGGACGTTTGGGTCTTCGA-3' \\
\hline & TCF3-sg-AS & 5'-TCGAAGACCCAAACGTCCCGCGGGGCCCGTGCGCCCGGTGCCGTCTTCCT-3' \\
\hline \multirow{2}{*}{ ASCL1 } & ASCL1-sg-S & 5'-AGGAAGACGGCACCGGAGCCGCTCGCTGCAGCAGCGGTTTGGGTCTTCGA-3' \\
\hline & ASCL1-sg-AS & 5'-TCGAAGACCCAAACCGCTGCTGCAGCGAGCGGCTCCGGTGCCGTCTTCCT-3' \\
\hline \multirow{2}{*}{ Ngn2 } & Ngn2-sg-S & 5'-AGGAAGACGGCACCGGCTGACAGGAGGAGGAGGCGGGTTTGGGTCTTCGA-3' \\
\hline & Ngn2-sg-AS & 5'-TCGAAGACCCAAACCCGCCTCCTCCTCCTGTCAGCCGGTGCCGTCTTCCT-3' \\
\hline \multirow{2}{*}{ Oct4 } & Oct4-sg-S & 5'-AGGAAGACGGCACCGGGGAAAACCGGGAGACACAACGTTTGGGTCTTCGA-3' \\
\hline & Oct4-sg-AS & 5'-TCGAAGACCCAAACGTTGTGTCTCCCGGTTTTCCCCGGTGCCGTCTTCCT-3' \\
\hline \multirow{2}{*}{ Nanog } & Nanog-sg-S & 5'-AGGAAGACGGCACCGGAGAGTAACCCAGACTAGGTGGTTTGGGTCTTCGA-3' \\
\hline & Nanog-sg-AS & 5'-TCGAAGACCCAAACCACCTAGTCTGGGTTACTCTCCGGTGCCGTCTTCCT-3' \\
\hline \multirow{2}{*}{ TNFAIP3 } & TNFAIP3-sg-S & 5'-AGGAAGACGGCACCGGCCGCCCCGCCCGGTCCCTGCGTTTGGGTCTTCGA-3' \\
\hline & TNFAIP3-sg-AS & 5'-TCGAAGACCCAAACGCAGGGACCGGGCGGGGCGGCCGGTGCCGTCTTCCT-3' \\
\hline \multirow{2}{*}{ CASP9 } & CASP9-sg-S & 5'-AGGAAGACGGCACCGGGGTGACGTGAGGTCAGTGCGGTTTGGGTCTTCGA-3' \\
\hline & CASP9-sg-AS & 5'-TCGAAGACCCAAACCGCACTGACCTCACGTCACCCCGGTGCCGTCTTCCT-3' \\
\hline \multirow{2}{*}{ CSF3 } & CSF3-sg-S & 5'-AGGAAGACGGCACCGGGGGAGGAAGGGAGTTTGAGGGTTTGGGTCTTCGA-3' \\
\hline & CSF3-sg-AS & 5'-TCGAAGACCCAAACCCTCAAACTCCCTTCCTCCCCCGGTGCCGTCTTCCT-3' \\
\hline \multirow{2}{*}{ Sox 2} & Sox2-sg-S & 5'-AGGAAGACGGCACCGGGGAGGAGGGGGCAGGCGAGGGTTTGGGTCTTCGA-3' \\
\hline & Sox2-sg-AS & 5'-TCGAAGACCCAAACCCTCGCCTGCCCCCTCCTCCCCGGTGCCGTCTTCCT-3' \\
\hline
\end{tabular}

S, sense; AS, antisense. 
Supplementary Table 3. Primers used as qPCR detection of gene expression (see Figure 5).

\begin{tabular}{|c|c|c|}
\hline Gene & Name & Sequence \\
\hline \multirow{2}{*}{ Hnf4a } & Hnf4a-F & 5'-GACATTCGGGCGAAGAAGAT-3' \\
\hline & Hnf4a-R & 5'-AAGATGATGGCTTTGAGGTAGG-3' \\
\hline \multirow{2}{*}{ TNFAIP3 } & TNFAIP3-F & 5'-TCATCCACAAAGCCCTCATC-3' \\
\hline & TNFAIP3-R & 5'-CATCATTCCAGTTCCGAGTATCA-3' \\
\hline \multirow{2}{*}{ TCF3 } & TCF3-F & 5'-ACGAGCGTATGGGCTACCA-3' \\
\hline & TCF3-R & 5'-GTTATTGCTTGAGTGATCCGGG-3' \\
\hline \multirow{2}{*}{ CSF3 } & CSF3-F & 5'-CCGACTCCTACAGTGGGCTA-3' \\
\hline & CSF3-R & 5'-CGCTGACGTGTTCTCCTCG-3' \\
\hline \multirow{2}{*}{ ASCL1 } & ASCL1-F & 5'-CGCGGCCAACAAGAAGATG-3' \\
\hline & ASCL1-R & 5'-CGACGAGTAGGATGAGACCG-3' \\
\hline \multirow{2}{*}{ Ngn2 } & Ngn2-F & 5'-AGGAAGAGGACGTGTTAGTGC-3' \\
\hline & Ngn2-R & 5'-GCAATCGTGTACCAGACCCAG-3' \\
\hline \multirow{2}{*}{ Sox 2} & Sox2-F & 5'-GTGAACCAGCGCATGGACAG-3' \\
\hline & Sox2-R & 5'-TCTGCGAGCTGGTCATGGAG-3' \\
\hline \multirow{2}{*}{ Oct4 } & Oct4-F & 5'-CTTGAATCCCGAATGGAAAGGG-3' \\
\hline & Oct4-R & 5'-GTGTATATCCCAGGGTGATCCTC-3' \\
\hline \multirow{2}{*}{ Nanog } & Nanog-F & 5'-CCTGGAACAGTCCCTTCTATAAC-3' \\
\hline & Nanog-R & 5'-TCACTCATCTTCACACGTCTTC-3' \\
\hline \multirow{2}{*}{ CASP9 } & CASP9-F & 5'-CGAACTAACAGGCAAGCAGCAAAG-3' \\
\hline & CASP9-R & 5'-AGAGCACCGACATCACCAAATCC-3' \\
\hline \multirow{2}{*}{ GAPDH } & GAPDH-F & 5'-GGATTTGGTCGTATTGGG-3' \\
\hline & GAPDH-R & 5'-GGAAGATGGTGATGGGATT-3' \\
\hline
\end{tabular}


Supplementary Table 4. Primers used as qPCR detection of gene expression (see Figure 6).

\begin{tabular}{|c|c|c|}
\hline Gene & Name & Sequence \\
\hline \multirow{2}{*}{ CD90 } & CD90-F & 5'-CGGAAGACCCCAGTCCA-3' \\
\hline & CD90-R & 5'-ACGAAGGCTCTGGTCCACTA-3' \\
\hline \multirow{2}{*}{ CD133 } & CD133-F & 5'-ACATGAAAAGACCTGGGGG-3' \\
\hline & CD133-R & 5'-GATCTGGTGT CCCAGCATG-3' \\
\hline \multirow{2}{*}{ Oct4 } & Oct4-F & 5'-CTTGAATCCCGAATGGAAAGGG-3' \\
\hline & Oct4-R & 5'-GTGTATATCCCAGGGTGATCCTC-3' \\
\hline \multirow{2}{*}{ Sox 2} & Sox $2-\mathrm{F}$ & 5'-GTGAACCAGCGCATGGACAG-3' \\
\hline & Sox $2-\mathrm{R}$ & 5'-TCTGCGAGCTGGTCATGGAG-3' \\
\hline \multirow{2}{*}{ Nanog } & Nanog-F & 5'-CCTGGAACAGTCCCTTCTATAAC-3' \\
\hline & Nanog-R & 5'-TCACTCATCTTCACACGTCTTC-3' \\
\hline \multirow{2}{*}{ LIN28 } & LIN28-F & 5'-TGTAAGTGGTTCAACGTGCG-3' \\
\hline & LIN28-R & 5'-CСТCACССТCСТTCAAGCTC-3' \\
\hline \multirow{2}{*}{$\mathrm{c}-\mathrm{Myc}$} & c-Myc-F & 5'-GCTGCTTAGACGCTGGATTT-3' \\
\hline & c-Myc-R & 5'-CTCCTCCTCGTCGCAGTAGA-3' \\
\hline \multirow{2}{*}{ Klf4 } & Klf4-F & 5'-GCGGCAAAACCTACACAAAG-3' \\
\hline & Klf4-R & 5'-CCCCGTGTGTTTACGGTAGT-3' \\
\hline \multirow{2}{*}{$\mathrm{P} 21$} & $\mathrm{P} 21-\mathrm{F}$ & 5'-GGATGTCCGTCAGAACCCAT-3' \\
\hline & $\mathrm{P} 21-\mathrm{R}$ & 5'-CCCTCCAGTGGTGTCTCGGTG-3' \\
\hline \multirow{2}{*}{ GS } & GS-F & 5'-CCTGCTTGTATGCTGGAGTC-3' \\
\hline & GS-R & 5'-GAAAAGTCGTTGATGTTGGA-3' \\
\hline \multirow{2}{*}{$\mathrm{BR}$} & BR-F & 5'-ACAAGGTGCTGCGGGAATCA-3' \\
\hline & BR-R & 5'-ACTGGTGGGAGGGGTAGGTG-3' \\
\hline \multirow{2}{*}{ ALDOB } & ALDOB-F & 5'-AGGAGGACTCTTCTCTCCCAA-3' \\
\hline & ALDOB-R & 5'-GATTCATCTGCAGCCAGGAT-3' \\
\hline \multirow{2}{*}{ CYP1 $1 \alpha 2$} & CYP1 $1 \alpha 2-F$ & 5'-CTGGCCTCTGCCATCTTCTG-3' \\
\hline & CYP1 $1 \alpha 2-R$ & 5'-TTAGCCTCCTTGCTCACATGC-3' \\
\hline \multirow{2}{*}{ PEPCK } & PEPCK-F & 5'-GTGTCCCTCTAGTCTATGAAGC-3' \\
\hline & PEPCK-R & 5'-ATTGACTTGATCCTCCAGATAC-3' \\
\hline \multirow{2}{*}{ APOCIII } & APOCIII-F & 5'-GGGTACTCCTTGTTGTTGC-3' \\
\hline & APOCIII-R & 5'-AAATCCCAGAACTCAGAGAAC-3' \\
\hline \multirow{2}{*}{ G-6-P } & G-6-P-F & 5'-GGCTCCATGACTGTGGGATC-3' \\
\hline & G-6-P-R & 5'-TTCAGCTGCACAGCCCAGAA-3' \\
\hline \multirow{2}{*}{$\mathrm{CPA} 2$} & CPA2-F & 5'-AGTGGGTTACACAAGCTACG-3' \\
\hline & CPA2-R & 5'-AGAGGCTTCCAGATACCTTG-3' \\
\hline \multirow{2}{*}{ CX32 } & CX32-F & 5'-CTGCAGACATTCTCTGGGAAA-3' \\
\hline & CX32-R & 5'-GCACCATGATTCTGAAGATGA-3' \\
\hline \multirow{2}{*}{ TP53INP1 } & TP53INP1-F & 5'-AAACCTTCTCATTGAACATCCC-3' \\
\hline & TP53INP1-R & 5'-CCATTGTGCTTGACTTGCC-3' \\
\hline \multirow{2}{*}{ MIST1 } & MIST1-F & 5'-CCAGCACTACCAGCAGCA-3' \\
\hline & MIST1-R & 5'-AGGACTGGGCGCTAGGTG-3' \\
\hline \multirow{2}{*}{ PRSS2 } & PRSS2-F & 5'-GTTGCAGCTGCTGTTGCTGCC-3' \\
\hline & PRSS2-R & 5'-TGTCATTGTCCAGAGTCCGGC-3' \\
\hline \multirow{2}{*}{ CELA3A } & CELA3A-F & 5'-CTCGTGTGTGGCCTGTGGCA-3' \\
\hline & CELA3A-R & 5'-CCAGAAACAAAGCTGGTCACA-3' \\
\hline
\end{tabular}




\section{Supplementary Figures}
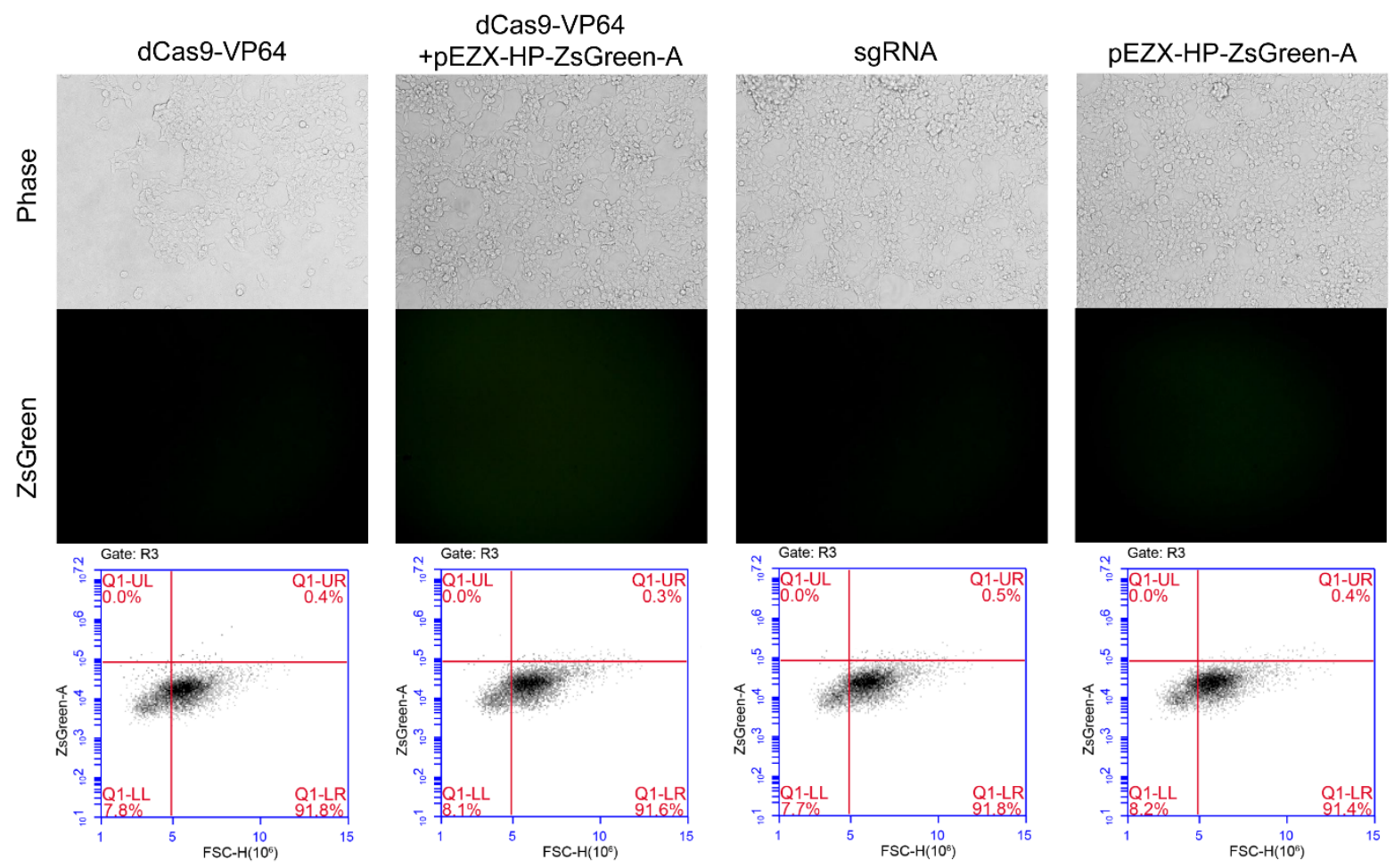

Supplementary Figure 1. Activation of reporter gene ZsGreen under the control of $H N F 4 \alpha$ promoter in HepG2 cells with various activators. Cells were photographed and the fluorescence intensity was quantified by flow cytometry. The figure showed the images and fluorescence quantification results of cells as negative control transfections of Fig.3. The reporter gene activation efficiency was indicated by the percentage of cells with green fluorescence over the threshold (cells in Q1-UR quadrant). 

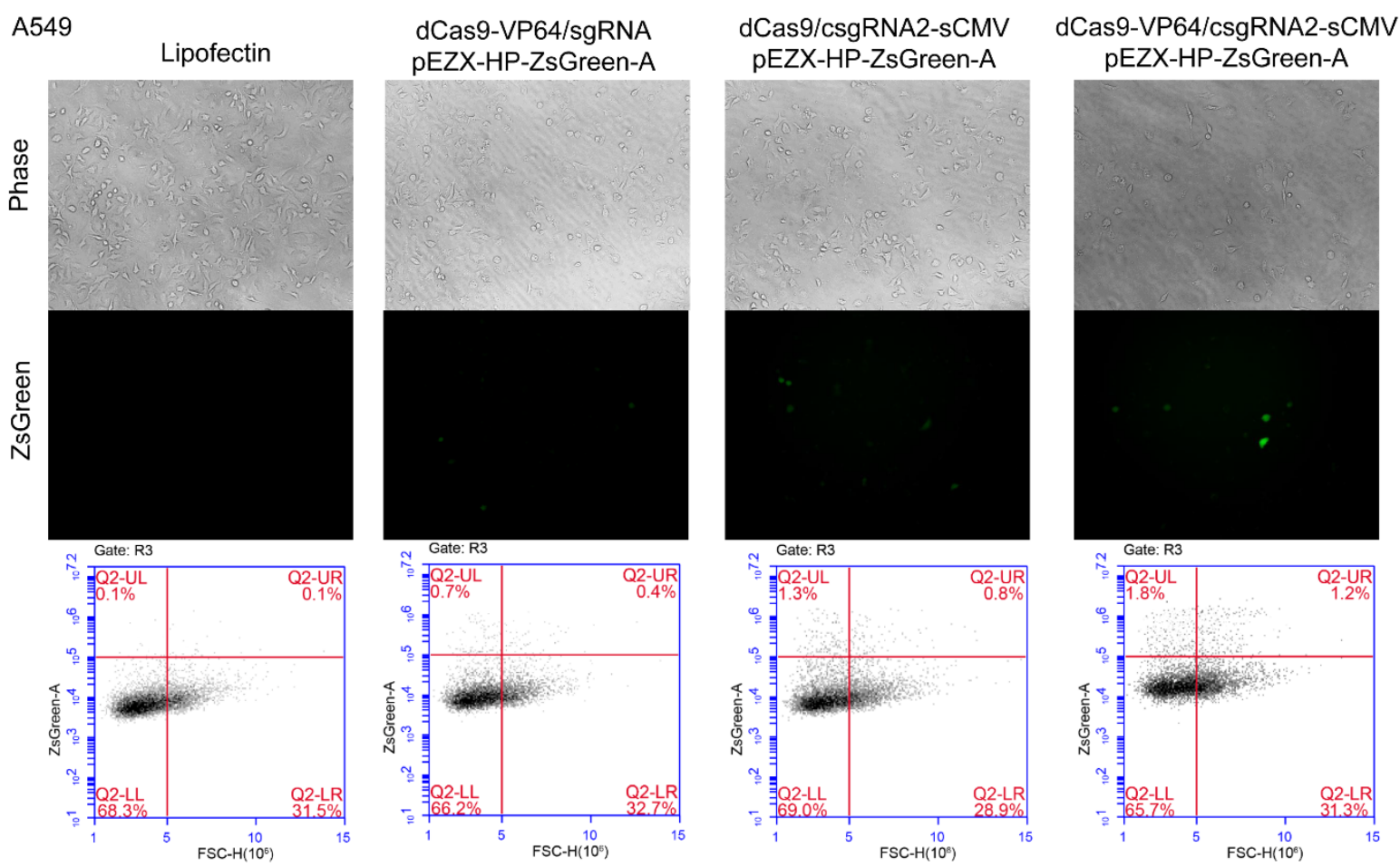

Supplementary Figure 2. Activation of an exogenous reporter gene ZsGreen under the control of a $H N F 4 \alpha$ promoter by the CRISPR-assistant trans enhancer in the A549 cells. Cells were transfected with three transcriptional activation systems, including dCas9-VP64 \& sgRNA, dCas9 \& csgRNA \& sCMV, and dCas9-VP64 \& csgRNA2 \& sCMV, together with a reporter construct (pEZX-HP-ZsGreen-A), respectively. Cells were photographed with a fluorescent microscope and their florescence intensity was analyzed by flow cytometry. The reporter gene activation efficiency was indicated by the percentage of cells with green fluorescence over the threshold (cells in Q1-UR quadrant). 

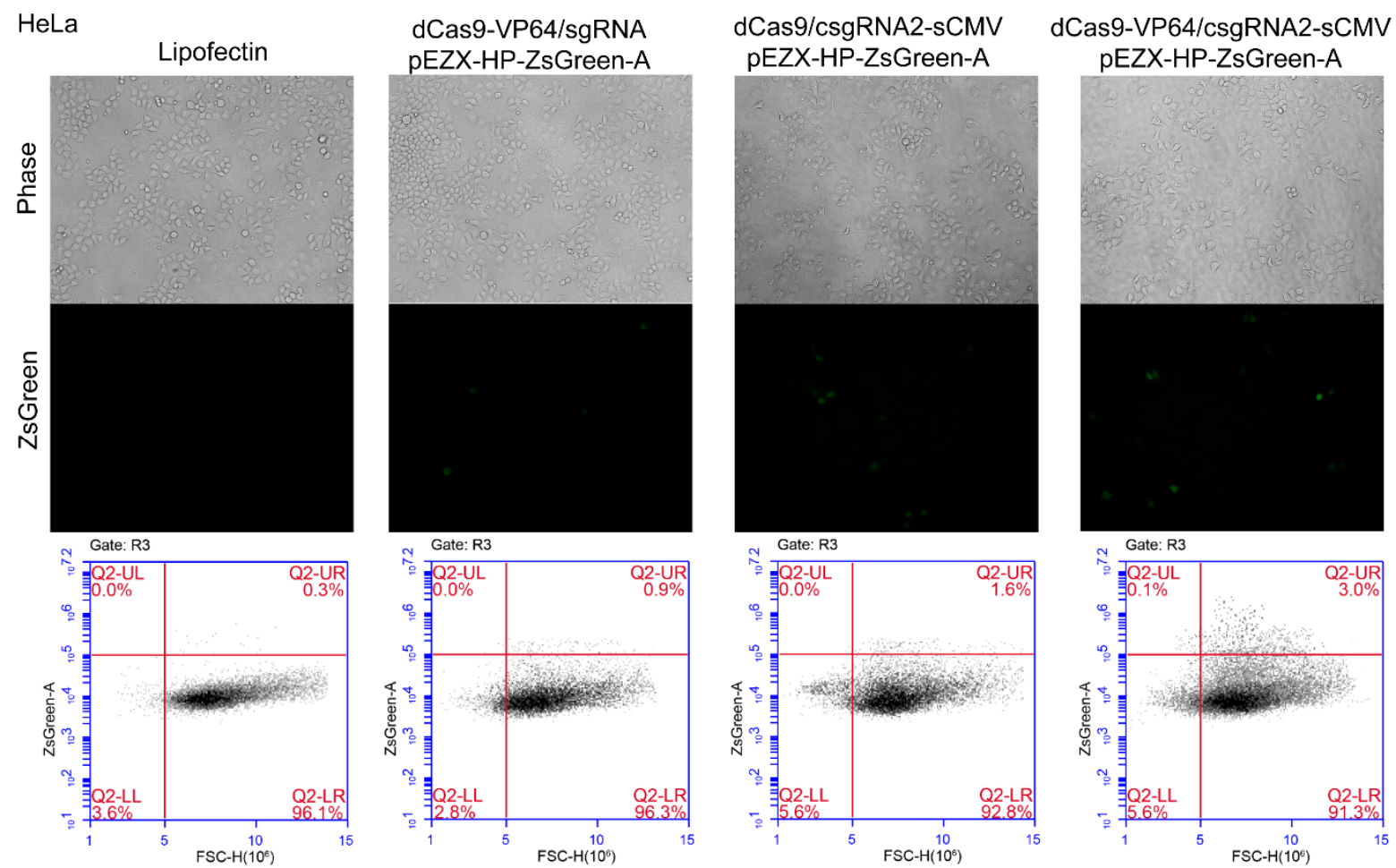

Supplementary Figure 3. Activation of an exogenous reporter gene ZsGreen under the control of a $H N F 4 \alpha$ promoter by the CRISPR-assistant trans enhancer in the HeLa cells. Cells were transfected with three transcriptional activation systems, including dCas9-VP64 \& sgRNA, dCas9 \& csgRNA \& sCMV, and dCas9-VP64 \& csgRNA2 \& sCMV, together with a reporter construct (pEZX-HP-ZsGreen-A), respectively. Cells were photographed with a fluorescent microscope and their florescence intensity was analyzed by flow cytometry. The reporter gene activation efficiency was indicated by the percentage of cells with green fluorescence over the threshold (cells in Q1-UR quadrant). 

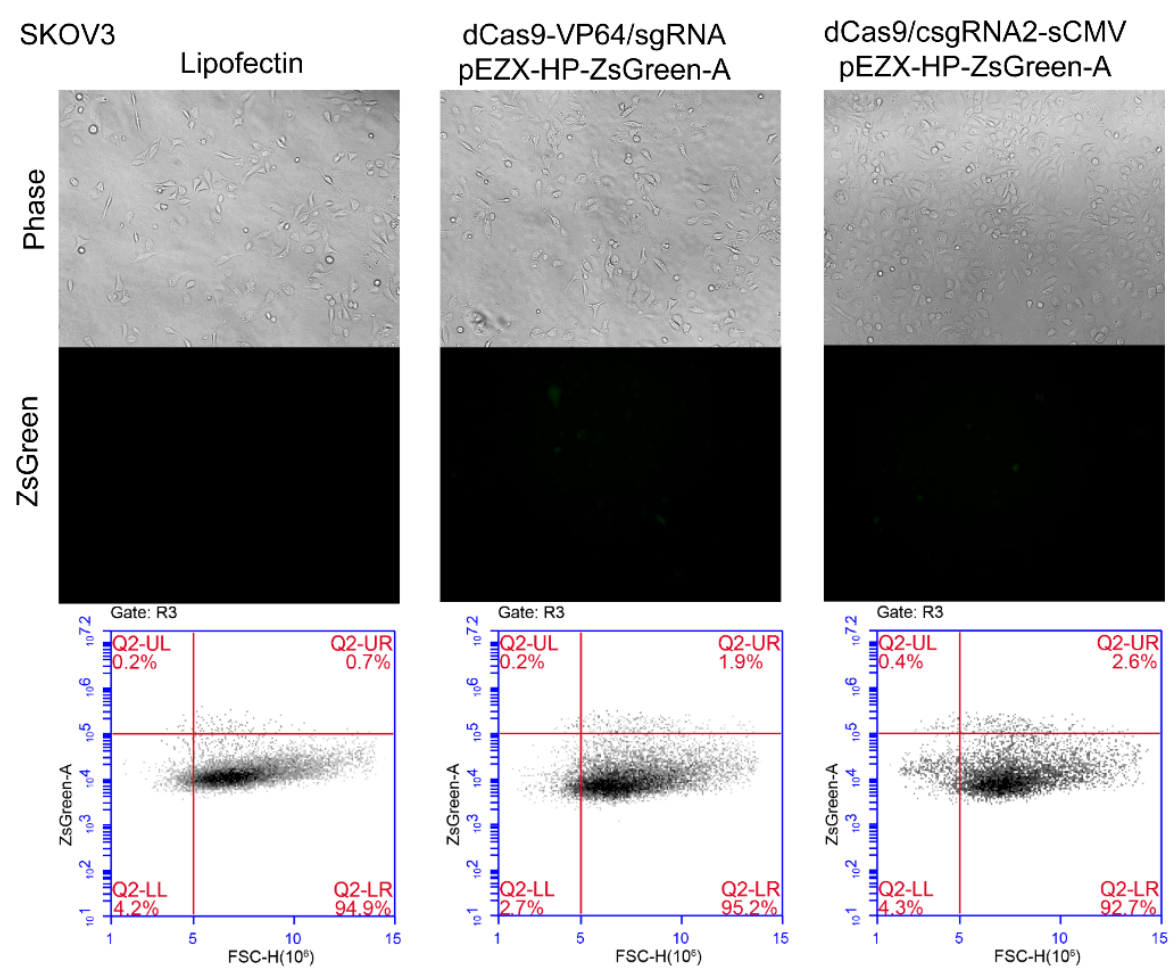

dCas9-VP64/csgRNA2-sCMV pEZX-HP-ZsGreen-A

Supplementary Figure 4. Activation of an exogenous reporter gene ZsGreen under the control of a $H N F 4 \alpha$ promoter by the CRISPR-assistant trans enhancer in the SKOV3 cells. Cells were transfected with three transcriptional activation systems, including dCas9-VP64 \& sgRNA, dCas9 \& csgRNA \& sCMV, and dCas9-VP64 \& csgRNA2 \& sCMV, together with a reporter construct (pEZX-HP-ZsGreen-A), respectively. Cells were photographed with a fluorescent microscope and their florescence intensity was analyzed by flow cytometry. The reporter gene activation efficiency was indicated by the percentage of cells with green fluorescence over the threshold (cells in Q1-UR quadrant). 

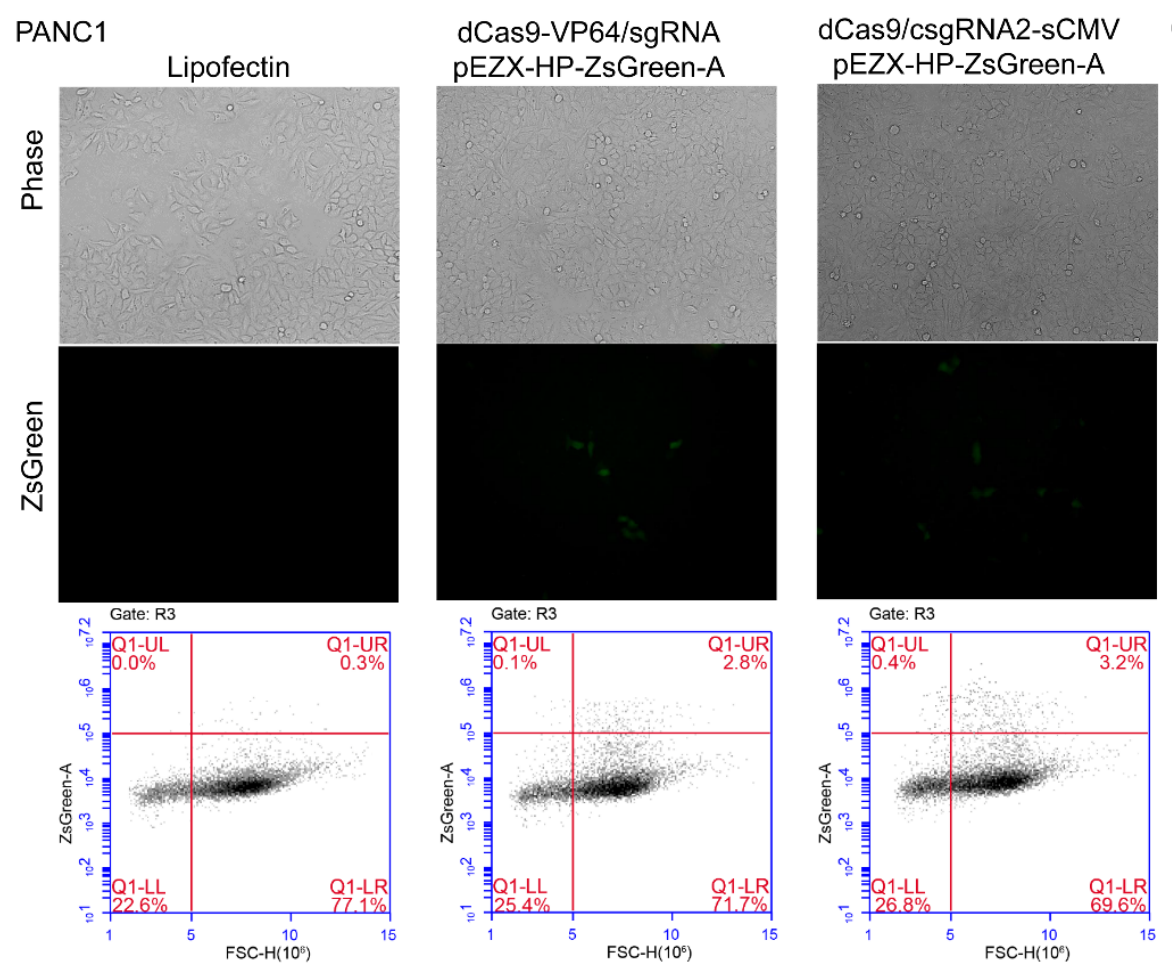

dCas9-VP64/csgRNA2-sCMV
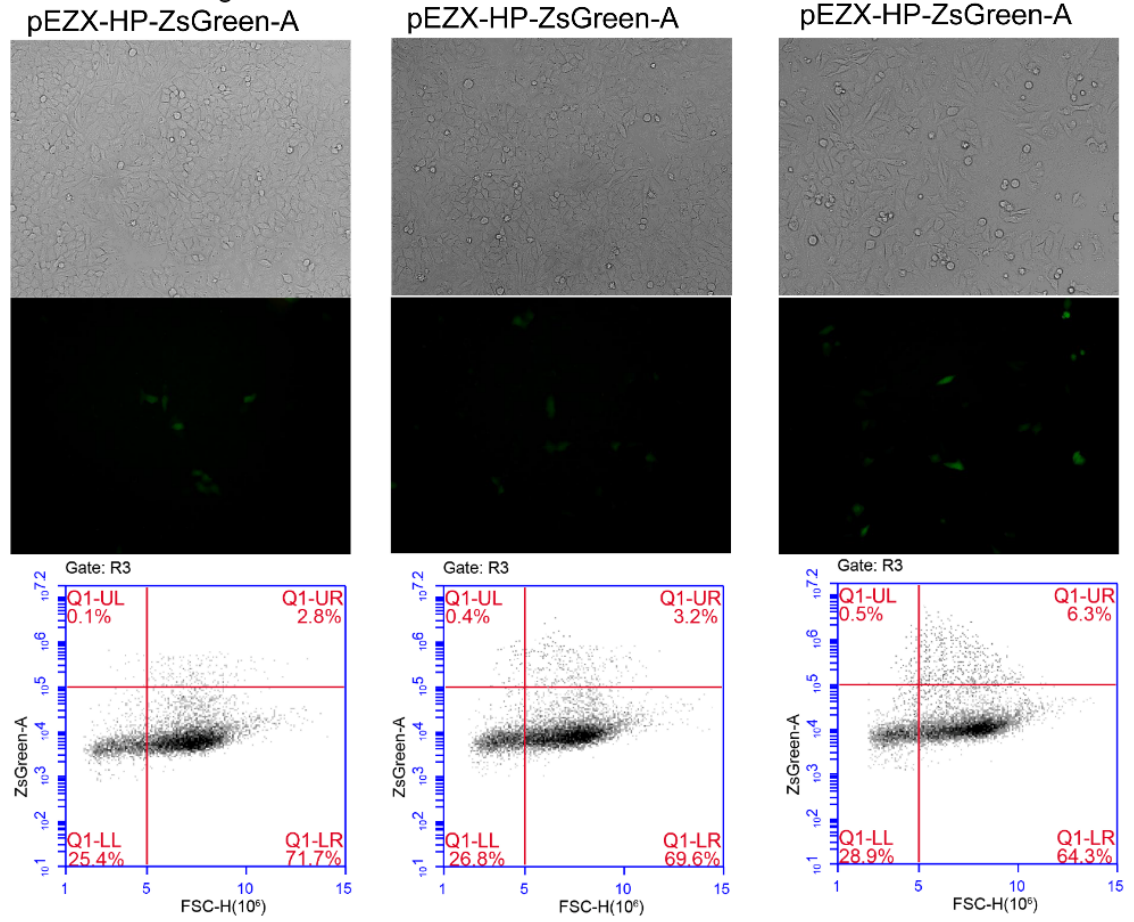

Supplementary Figure 5. Activation of an exogenous reporter gene ZsGreen under the control of a HNF4 $\alpha$ promoter by the CRISPR-assistant trans enhancer in the PANC1 cells. Cells were transfected with three transcriptional activation systems, including dCas9-VP64 \& sgRNA, dCas9 \& csgRNA \& sCMV, and dCas9-VP64 \& csgRNA2 \& sCMV, together with a reporter construct (pEZX-HP-ZsGreen-A), respectively. Cells were photographed with a fluorescent microscope and their florescence intensity was analyzed by flow cytometry. The reporter gene activation efficiency was indicated by the percentage of cells with green fluorescence over the threshold (cells in Q1-UR quadrant). 

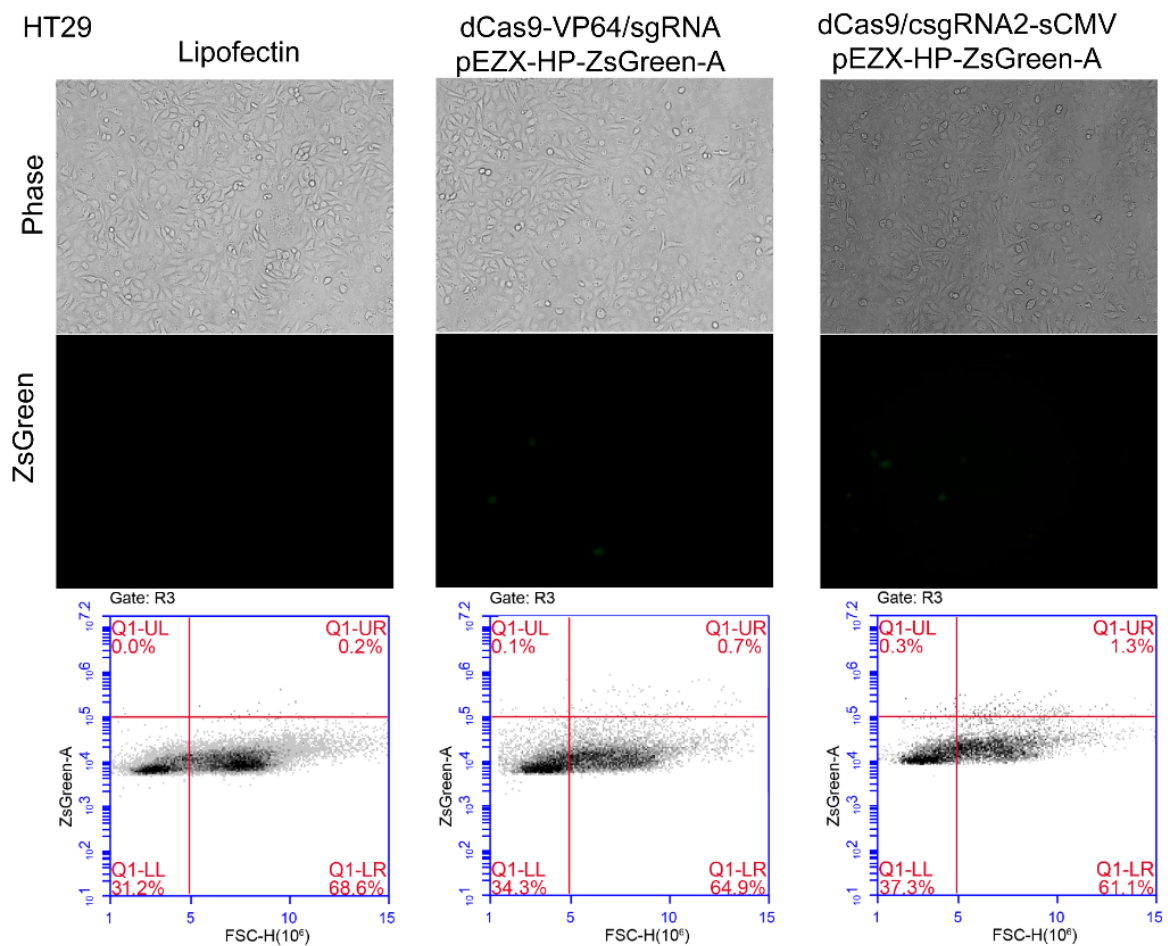

dCas9-VP64/csgRNA2-sCMV
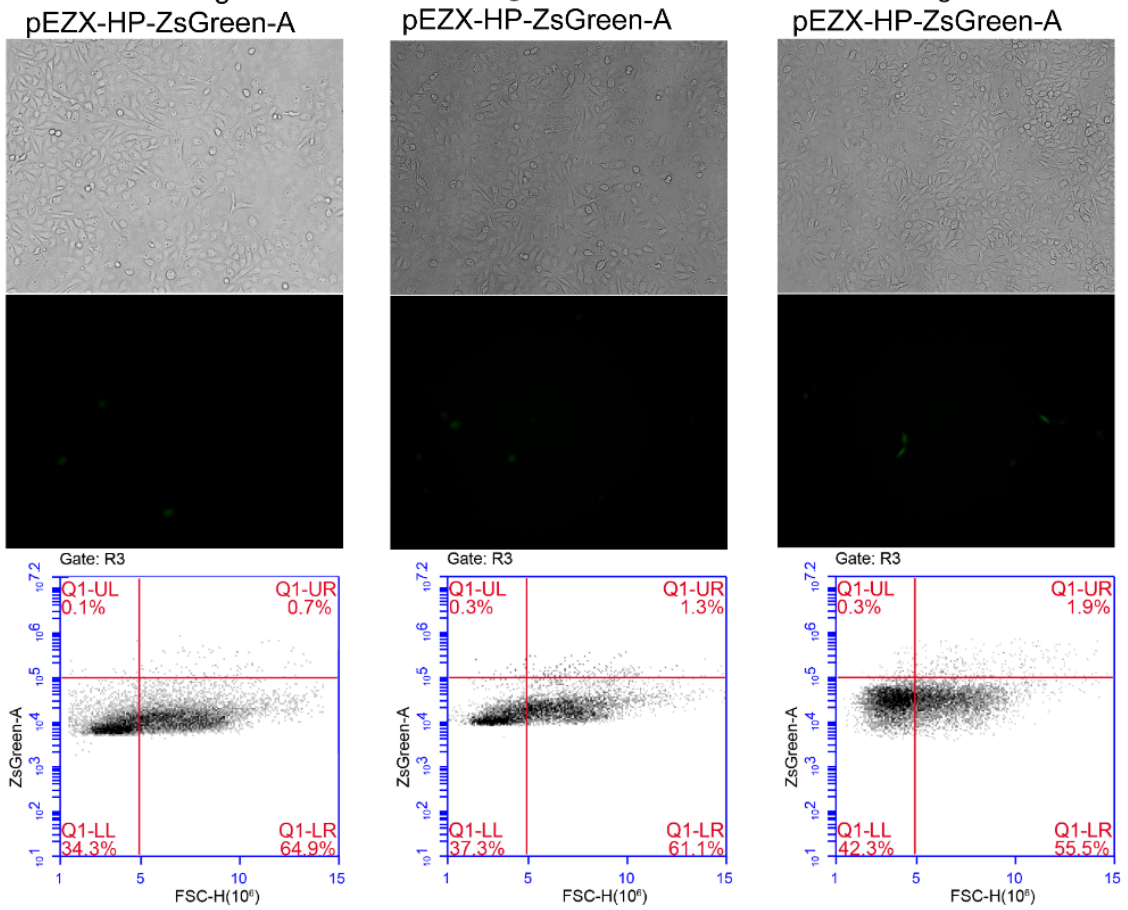

Supplementary Figure 6. Activation of an exogenous reporter gene ZsGreen under the control of a HNF4 $\alpha$ promoter by the CRISPR-assistant trans enhancer in the HT29 cells. Cells were transfected with three transcriptional activation systems, including dCas9-VP64 \& sgRNA, dCas9 \& csgRNA \& sCMV, and dCas9-VP64 \& csgRNA2 \& sCMV, together with a reporter construct (pEZX-HP-ZsGreen-A), respectively. Cells were photographed with a fluorescent microscope and their florescence intensity was analyzed by flow cytometry. The reporter gene activation efficiency was indicated by the percentage of cells with green fluorescence over the threshold (cells in Q1-UR quadrant). 

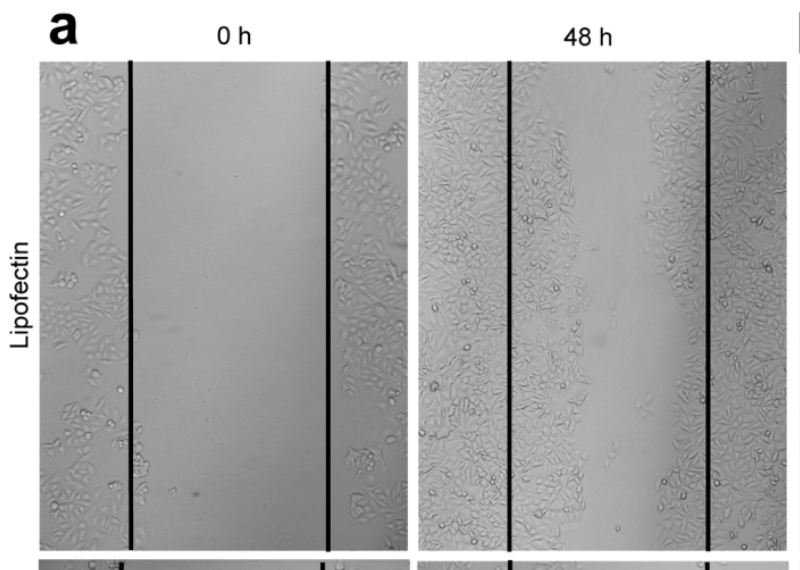

b $\quad 0 \mathrm{~h}$
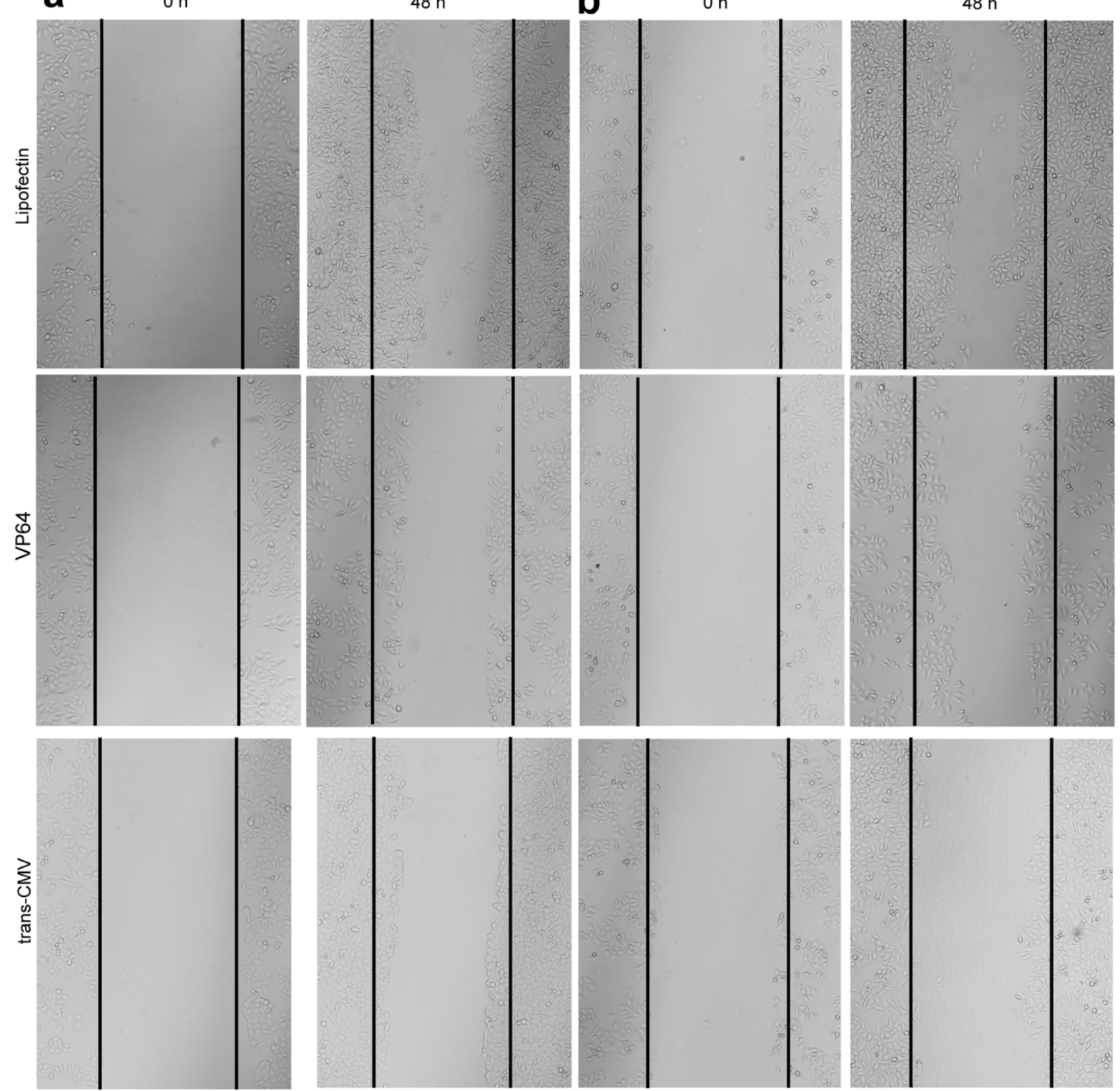

Supplementary Figure 7. The wound-healing assay. Cells were transfected with dCas9-VP64/csgRNA (VP64) and dCas9-VP64/csgRNA\& sCMV (Trans-CMV) to activate endogenous genes $H N F 4 \alpha$ in HepG2 cell and $E 47$ in in PANC-1 cell. a. The transferred HepG2 cells. b. The transferred PANC-1 cells. 

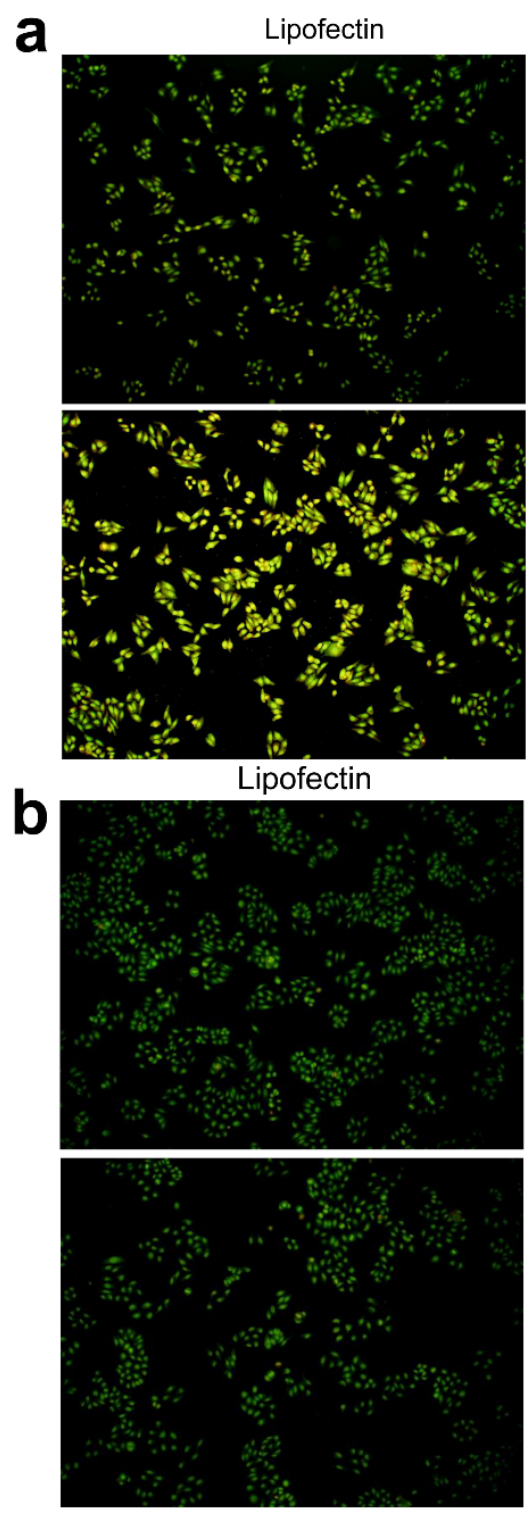

C
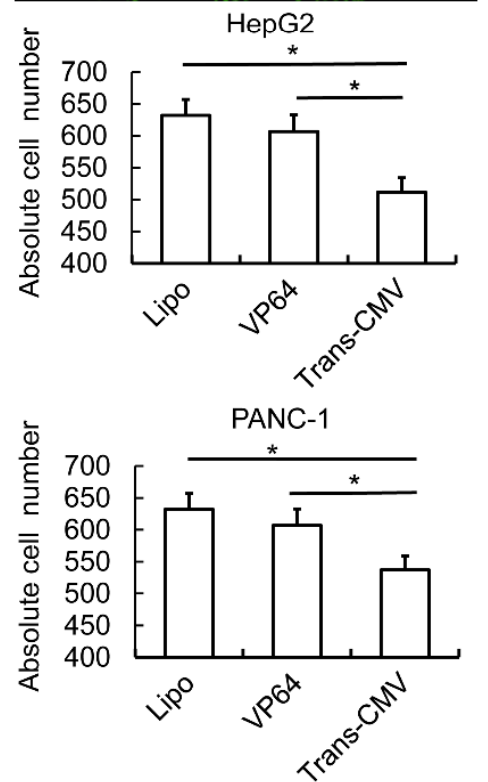

VP64
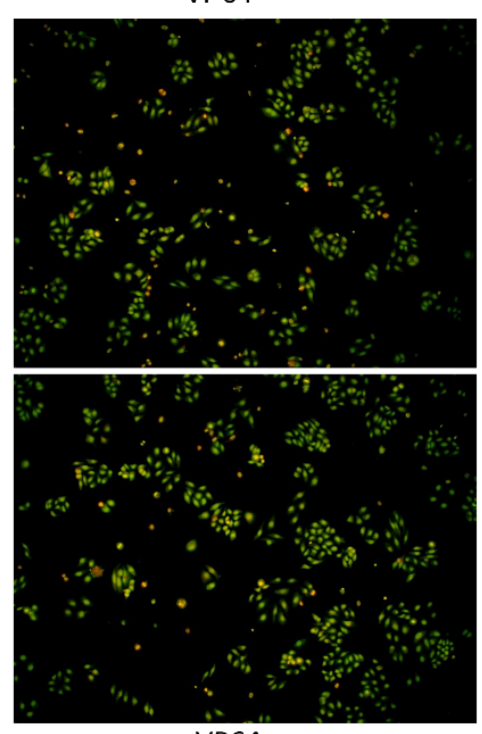

VP64
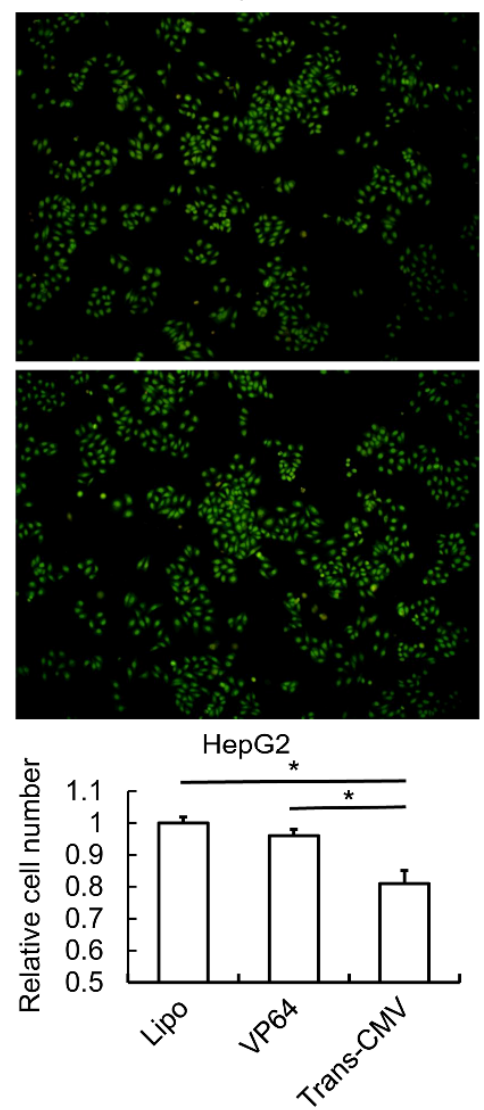

PANC-1

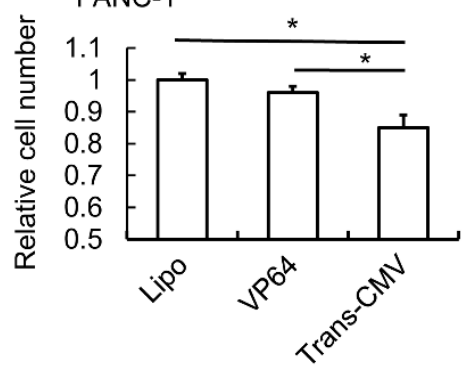

Trans-CMV

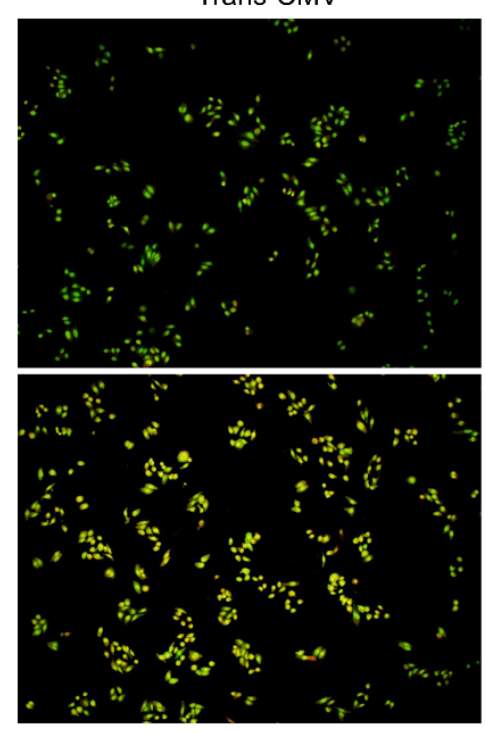

Trans-CMV

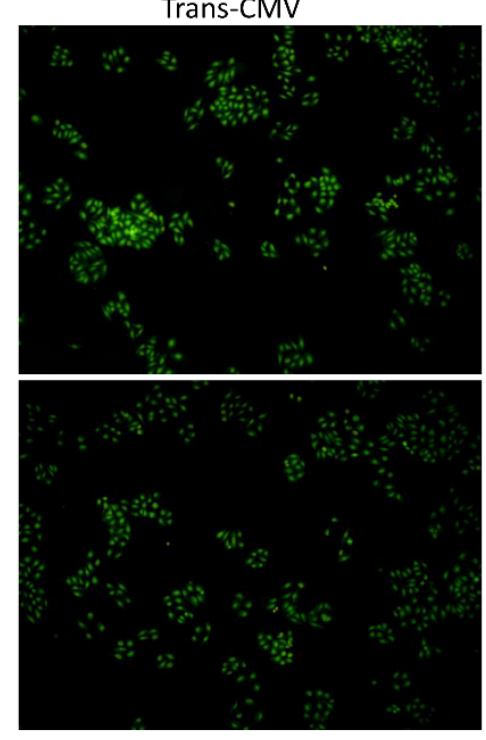

HepG2

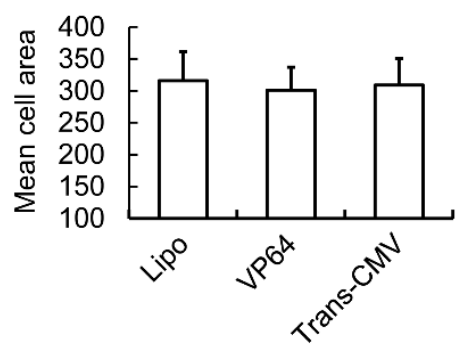

PANC-1

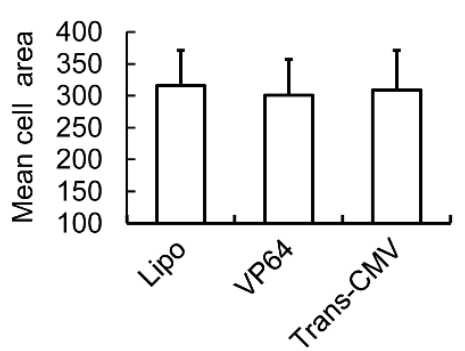

Supplementary Figure 8. The transwell assay. Cells were transfected with dCas9-VP64/csgRNA (VP64) 
bioRxiv preprint doi: https://doi.org/10.1101/517920; this version posted January 11,2019 . The copyright holder for this preprint (which was not certified by peer review) is the author/funder. All rights reserved. No reuse allowed without permission.

and dCas9-VP64/csgRNA\& sCMV (Trans-CMV) to activate endogenous genes HNF4 $\alpha$ in HepG2 cell and E47 in in PANC-1 cell. a. The transferred HepG2 cells. b. The transferred PANC-1 cells. c. Counting of cell numbers. The images of acridine orange-stained cells were counted with ImageJ software. ${ }^{*}, p<0.05$. 
Transfection 1

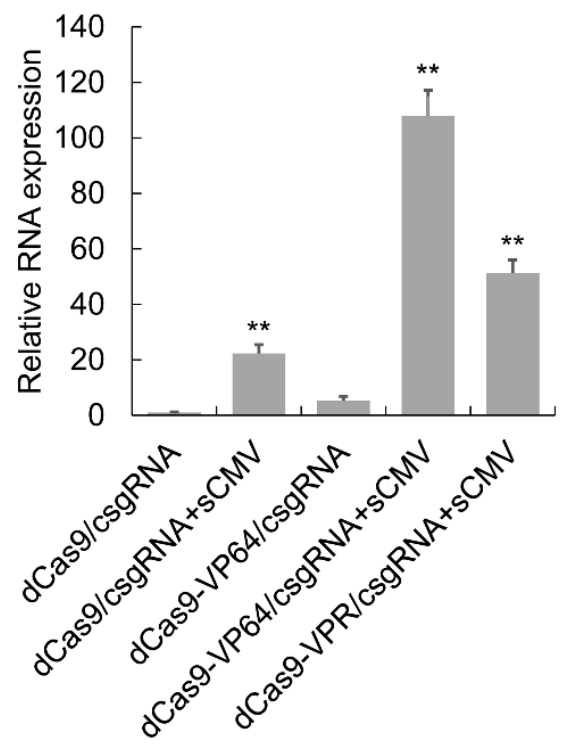

Transfection 2

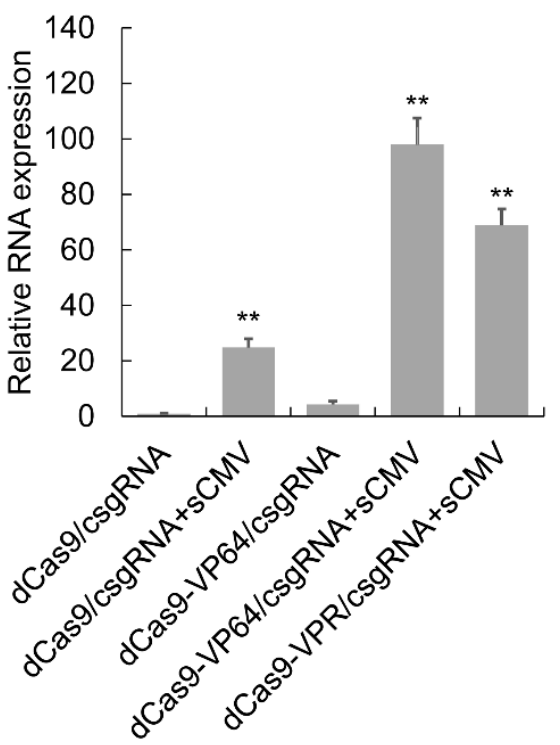

Supplementary Figure 9. Activation of endogenous $H N F 4 \alpha$ gene in $293 \mathrm{~T}$ cell with various systems. The $293 \mathrm{~T}$ cell was transfected with various systems to activate the endogenous $H N F 4 \alpha$ gene. The $H N F 4 \alpha$ and $\beta$-action genes were detected with qPCR. The HNF4 $\alpha$ expression level was showed as the fold of $\beta$-action. 
a

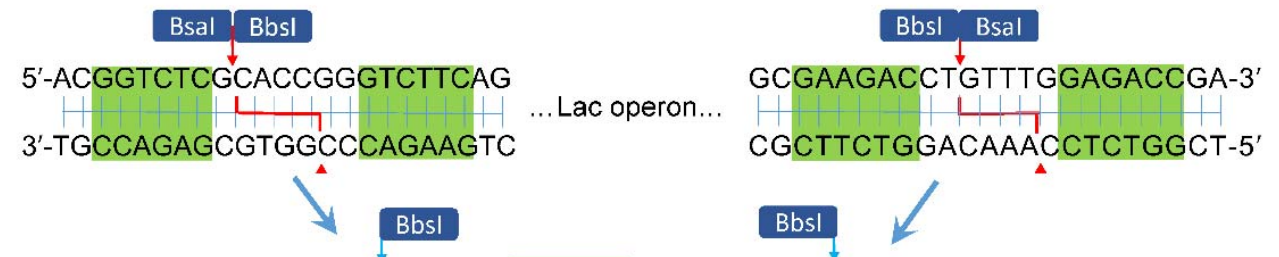

GGAAAGGACGAAACACCGGGTCTTCGAGAAGACCTGTTTTAGAGCTAGAAA

CCTTTCCTGCTTTTGTGGCCCAGAAGCTCTTCTGGACAAAATCTCGATCTTT px458

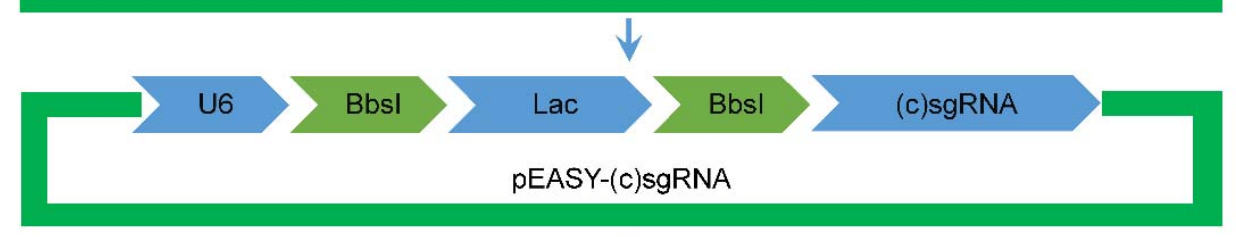

b

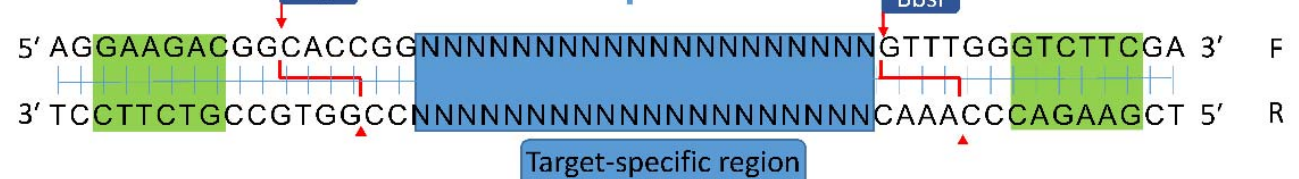

Target-specific region

Supplementary Figure 10. Construction of sgRNA vectors for blue-white screening. 


\section{Supplementary sequences}

\section{Sequences of vectors, templates, sgRNA, csgRNA, and sCMV:}

Receptor of pEASY-U6-csgRNA-1:

U6 promoter + Bbsl site + lac operon + Bbsl site + gRNA scaffold + Flanking sequence 1 GAGGGCCTATTTCCCATGATTCCTTCATATTTGCATATACGATACAAGGCTGTTAGAGAGATAA TTGGAATTAATTTGACTGTAAACACAAAGATATTAGTACAAAATACGTGACGTAGAAAGTAATAA TTTCTTGGGTAGTTTGCAGTTTTAAAATTATGTTTTAAAATGGACTATCATATGCTTACCGTAAC TTGAAAGTATTTCGATTTCTTGGCTTTATATATCTTGTGGAAAGGACGAAACACCGGGTCTTCA GCGCCCAATACGCAAACCGCCTCTCCCCGCGCGTTGGCCGATTCATTAATGCAGCTGGCAC GACAGGTTTCCCGACTGGAAAGCGGGCAGTGAGCGCAACGCAATTAATGTGAGTTAGCTCA CTCATTAGGCACCCCAGGCTTTACACTTTATGCTTCCGGCTCGTATGTTGTGTGGAATTGTGA GCGGATAACAATTTCACACAGGAAACAGCTATGACCATGATTACGCCAAGCTGCCCTTAAGG GCAGCTTCAATTCGCCCTATAGTGAGTCGTATTACAATTCACTGGCCGTCGTTTTACAACGTC GTGACTGGGAAAACCCTGGCGTTACCCAACTTAATCGCCTTGCAGCACATCCCCCTTTCGCC AGCTGGCGTAATAGCGAAGAGGCCCGCACCGATCGCCCTTCCCAACAGTTGCGCAGCCTGA ATGGCGAATGGACGCGAAGACCTGTTTTAGAGCTAGAAATAGCAAGTTAAAATAAGGCTAGTC CGTTATCAACTTGAAAAAGTGGCACCGAGTCGGTGCAATCGGGCCGACGGCAAACATACCTT TTTT

Receptor of pEASY-U6-csgRNA-2:

U6 promoter + Bbsl site + lac operon + Bbsl site + gRNA scaffold + Flanking sequence 2 GAGGGCCTATTTCCCATGATTCCTTCATATTTGCATATACGATACAAGGCTGTTAGAGAGATAA TTGGAATTAATTTGACTGTAAACACAAAGATATTAGTACAAAATACGTGACGTAGAAAGTAATAA TTTCTTGGGTAGTTTGCAGTTTTAAAATTATGTTTTAAAATGGACTATCATATGCTTACCGTAAC TTGAAAGTATTTCGATTTCTTGGCTTTATATATCTTGTGGAAAGGACGAAACACCGGGTCTTCA GCGCCCAATACGCAAACCGCCTCTCCCCGCGCGTTGGCCGATTCATTAATGCAGCTGGCAC GACAGGTTTCCCGACTGGAAAGCGGGCAGTGAGCGCAACGCAATTAATGTGAGTTAGCTCA CTCATTAGGCACCCCAGGCTTTACACTTTATGCTTCCGGCTCGTATGTTGTGTGGAATTGTGA GCGGATAACAATTTCACACAGGAAACAGCTATGACCATGATTACGCCAAGCTGCCCTTAAGG GCAGCTTCAATTCGCCCTATAGTGAGTCGTATTACAATTCACTGGCCGTCGTTTTACAACGTC GTGACTGGGAAAACCCTGGCGTTACCCAACTTAATCGCCTTGCAGCACATCCCCCTTTCGCC AGCTGGCGTAATAGCGAAGAGGCCCGCACCGATCGCCCTTCCCAACAGTTGCGCAGCCTGA ATGGCGAATGGACGCGAAGACCTGTTTTAGAGCTAGAAATAGCAAGTTAAAATAAGGCTAGTC CGTTATCAACTTGAAAAAGTGGCACCGAGTCGGTGCGGAACCTTACGAATACCAGATGCTTT TTTT

Receptor of pEASY-U6-csgRNA-3:

U6 promoter + Bbsl site + lac operon + Bbsl site + gRNA scaffold + Flanking sequence 3 GAGGGCCTATTTCCCATGATTCCTTCATATTTGCATATACGATACAAGGCTGTTAGAGAGATAA TTGGAATTAATTTGACTGTAAACACAAAGATATTAGTACAAAATACGTGACGTAGAAAGTAATAA TTTCTTGGGTAGTTTGCAGTTTTAAAATTATGTTTTAAAATGGACTATCATATGCTTACCGTAAC TTGAAAGTATTTCGATTTCTTGGCTTTATATATCTTGTGGAAAGGACGAAACACCGGGTCTTCA 
GCGCCCAATACGCAAACCGCCTCTCCCCGCGCGTTGGCCGATTCATTAATGCAGCTGGCAC GACAGGTTTCCCGACTGGAAAGCGGGCAGTGAGCGCAACGCAATTAATGTGAGTTAGCTCA CTCATTAGGCACCCCAGGCTTTACACTTTATGCTTCCGGCTCGTATGTTGTGTGGAATTGTGA GCGGATAACAATTTCACACAGGAAACAGCTATGACCATGATTACGCCAAGCTGCCCTTAAGG GCAGCTTCAATTCGCCCTATAGTGAGTCGTATTACAATTCACTGGCCGTCGTTTTACAACGTC GTGACTGGGAAAACCCTGGCGTTACCCAACTTAATCGCCTTGCAGCACATCCCCCTTTCGCC AGCTGGCGTAATAGCGAAGAGGCCCGCACCGATCGCCCTTCCCAACAGTTGCGCAGCCTGA ATGGCGAATGGACGCGAAGACCTGTTTTAGAGCTAGAAATAGCAAGTTAAAATAAGGCTAGTC CGTTATCAACTTGAAAAAGTGGCACCGAGTCGGTGCATCTAGTGGAACCTCAAACATACCTTT TTT

Transcription template of normal sgRNA :

U6 promoter + target sequence + gRNA scaffold GAGGGCCTATTTCCCATGATTCCTTCATATTTGCATATACGATACAAGGCTGTTAGAGAGATAA TTGGAATTAATTTGACTGTAAACACAAAGATATTAGTACAAAATACGTGACGTAGAAAGTAATAA TTTCTTGGGTAGTTTGCAGTTTTAAAATTATGTTTTAAAATGGACTATCATATGCTTACCGTAAC TTGAAAGTATTTCGATTTCTTGGCTTTATATATCTTGTGGAAAGGACGAAACACCGGNNNNNN NNNNNNNNNNNNNNGTTTTAGAGCTAGAAATAGCAAGTTAAAATAAGGCTAGTCCGTTATCAA CTTGAAAAAGTGGCACCGAGTCGGTGCTTTTTT

Transcription template of csgRNA-1:

U6 promoter + target sequence + gRNA scaffold + Flanking sequence 1

GAGGGCCTATTTCCCATGATTCCTTCATATTTGCATATACGATACAAGGCTGTTAGAGAGATAA TTGGAATTAATTTGACTGTAAACACAAAGATATTAGTACAAAATACGTGACGTAGAAAGTAATAA TTTCTTGGGTAGTTTGCAGTTTTAAAATTATGTTTTAAAATGGACTATCATATGCTTACCGTAAC TTGAAAGTATTTCGATTTCTTGGCTTTATATATCTTGTGGAAAGGACGAAACACCGGNNNNNN NNNNNNNNNNNNNNGTTTTAGAGCTAGAAATAGCAAGTTAAAATAAGGCTAGTCCGTTATCAA CTTGAAAAAGTGGCACCGAGTCGGTGCAATCGGGCCGACGGCAAACATACCTTTTTT

Transcription template of csgRNA-2:

U6 promoter + target sequence + gRNA scaffold + Flanking sequence 2

GAGGGCCTATTTCCCATGATTCCTTCATATTTGCATATACGATACAAGGCTGTTAGAGAGATAA TTGGAATTAATTTGACTGTAAACACAAAGATATTAGTACAAAATACGTGACGTAGAAAGTAATAA TTTCTTGGGTAGTTTGCAGTTTTAAAATTATGTTTTAAAATGGACTATCATATGCTTACCGTAAC TTGAAAGTATTTCGATTTCTTGGCTTTATATATCTTGTGGAAAGGACGAAACACCGGNNNNNN NNNNNNNNNNNNNNGTTTTAGAGCTAGAAATAGCAAGTTAAAATAAGGCTAGTCCGTTATCAA CTTGAAAAAGTGGCACCGAGTCGGTGCGGAACCTTACGAATACCAGATGCTTTTTTT

Transcription template of csgRNA-3:

U6 promoter + target sequence + gRNA scaffold + Flanking sequence 3 GAGGGCCTATTTCCCATGATTCCTTCATATTTGCATATACGATACAAGGCTGTTAGAGAGATAA TTGGAATTAATTTGACTGTAAACACAAAGATATTAGTACAAAATACGTGACGTAGAAAGTAATAA TTTCTTGGGTAGTTTGCAGTTTTAAAATTATGTTTTAAAATGGACTATCATATGCTTACCGTAAC TTGAAAGTATTTCGATTTCTTGGCTTTATATATCTTGTGGAAAGGACGAAACACCGGNNNNNN 
NNNNNNNNNNNNNNGTTTTAGAGCTAGAAATAGCAAGTTAAAATAAGGCTAGTCCGTTATCAA CTTGAAAAAGTGGCACCGAGTCGGTGCATCTAGTGGAACCTCAAACATACCTTTTTT

\section{sgRNA:}

target sequence + gRNA scaffold

NNNNNNNNNNNNNNNNNNNNGUUUUAGAGCUAGAAAUAGCAAGUUAAAAUAAGGCUAGUC CGUUAUCAACUUGAAAAAGUGGCACCGAGUCGGUGCUUUU

\section{csgRNA-1:}

target sequence + gRNA scaffold + Capture sequence 1

NNNNNNNNNNNNNNNNNNNNGUUUUAGAGCUAGAAAUAGCAAGUUAAAAUAAGGCUAGUC CGUUAUCAACUUGAAAAAGUGGCACCGAGUCGGUGCAAUCGGGCCGACGGCAAACAUAC CUUUU

csgRNA-2:

target sequence + gRNA scaffold + Capture sequence 2

NNNNNNNNNNNNNNNNNNNNGUUUUAGAGCUAGAAAUAGCAAGUUAAAAUAAGGCUAGUC CGUUAUCAACUUGAAAAAGUGGCACCGAGUCGGUGCGGAACCUUACGAAUACCAGAUGC UUUUU

csgRNA-3:

target sequence + gRNA scaffold + Capture sequence 3

NNNNNNNNNNNNNNNNNNNNGUUUUAGAGCUAGAAAUAGCAAGUUAAAAUAAGGCUAGUC CGUUAUCAACUUGAAAAAGUGGCACCGAGUCGGUGCAUCUAGUGGAACCUCAAACAUAC CUUUU

SCMV-1:

CMV enhancer + CMV promoter + Nt.BbvCl site + Flanking sequence 1

5'-TAGTTATTAATAGTAATCAATTACGGGGTCATTAGTTCATAGCCCATATATGGAGTTCCGCGT TACATAACTTACGGTAAATGGCCCGCCTGGCTGACCGCCCAACGACCCCCGCCCATTGACG TCAATAATGACGTATGTTCCCATAGTAACGCCAATAGGGACTTTCCATTGACGTCAATGGGTG GAGTATTTACGGTAAACTGCCCACTTGGCAGTACATCAAGTGTATCATATGCCAAGTACGCCC CCTATTGACGTCAATGACGGTAAATGGCCCGCCTGGCATTATGCCCAGTACATGACCTTATGG GACTTTCCTACTTGGCAGTACATCTACGTATTAGTCATCGCTATTACCATGGTGATGCGGTTTT GGCAGTACATCAATGGGCGTGGATAGCGGTTTGACTCACGGGGATTTCCAAGTCTCCACCC CATTGACGTCAATGGGAGTTTGTTTTGGCACCAAAATCAACGGGACTTTCCAAAATGTCGTAA CAACTCCGCCCCATTGACGCAAATGGGCGGTAGGCGTGTACGGTGGGAGGTCTATATAAGC AGAGCTGCTGAGGGGTATGTTTGCCGTCGGCCCGATT

SCMV-2:

$\mathrm{CMV}$ enhancer + CMV promoter + Nt.BbvCl site + Flanking sequence 2

TAGTTATTAATAGTAATCAATTACGGGGTCATTAGTTCATAGCCCATATATGGAGTTCCGCGTTA CATAACTTACGGTAAATGGCCCGCCTGGCTGACCGCCCAACGACCCCCGCCCATTGACGTC AATAATGACGTATGTTCCCATAGTAACGCCAATAGGGACTTTCCATTGACGTCAATGGGTGGA 
GTATTTACGGTAAACTGCCCACTTGGCAGTACATCAAGTGTATCATATGCCAAGTACGCCCCC TATTGACGTCAATGACGGTAAATGGCCCGCCTGGCATTATGCCCAGTACATGACCTTATGGGA CTTTCCTACTTGGCAGTACATCTACGTATTAGTCATCGCTATTACCATGGTGATGCGGTTTTGG CAGTACATCAATGGGCGTGGATAGCGGTTTGACTCACGGGGATTTCCAAGTCTCCACCCCAT TGACGTCAATGGGAGTTTGTTTTGGCACCAAAATCAACGGGACTTTCCAAAATGTCGTAACA ACTCCGCCCCATTGACGCAAATGGGCGGTAGGCGTGTACGGTGGGAGGTCTATATAAGCAG AGCTGCTGAGGAGCATCTGGTATTCGTAAGGTTCC

SCMV-3:

$\mathrm{CMV}$ enhancer + CMV promoter + Nt.BbvCl site + Flanking sequence 3

TAGTTATTAATAGTAATCAATTACGGGGTCATTAGTTCATAGCCCATATATGGAGTTCCGCGTTA CATAACTTACGGTAAATGGCCCGCCTGGCTGACCGCCCAACGACCCCCGCCCATTGACGTC AATAATGACGTATGTTCCCATAGTAACGCCAATAGGGACTTTCCATTGACGTCAATGGGTGGA GTATTTACGGTAAACTGCCCACTTGGCAGTACATCAAGTGTATCATATGCCAAGTACGCCCCC TATTGACGTCAATGACGGTAAATGGCCCGCCTGGCATTATGCCCAGTACATGACCTTATGGGA CTTTCCTACTTGGCAGTACATCTACGTATTAGTCATCGCTATTACCATGGTGATGCGGTTTTGG CAGTACATCAATGGGCGTGGATAGCGGTTTGACTCACGGGGATTTCCAAGTCTCCACCCCAT TGACGTCAATGGGAGTTTGTTTTGGCACCAAAATCAACGGGACTTTCCAAAATGTCGTAACA ACTCCGCCCCATTGACGCAAATGGGCGGTAGGCGTGTACGGTGGGAGGTCTATATAAGCAG AGCTGCTGAGGGGTATGTTTGAGGTTCCACTAGAT

HNF4a promoter report vector:

HNF4a promoter + minimal TATA-box promoter + ZsGreen

TGAGATCCAAAACTGAGACAAAAGAAACGGGGCTGTTCCAAAAAAAAAGCTAGGTGGCAGG TGTCTAACATGCCAGGGAGCTAAAACAGAGTGTGTGAGTTTCAGCAGCAGGTTGAATTTAGA ATGGGGAAGGAGACCAGAGGAGACGCCAGACAGGATGACTTTGTCCCATTGGCCTGGAGG CAGCCCCATGTTTCTCCACCCCTCATATCACTCACCAGTTTGTAATAGTATCTTTGAATGACGA TCTGATTAAGGTCCGTCTCCTCCATTAGTCCACAAGTTTCGGGGGTACATCTACTTTGCTCAT TTCCATATCCCCAGAGTCTAGCACAAGGCCTGGTACATAGTAGGTGCTCAATAAATATGTTAGA TGAAAGGAAGATAACACCTCTATGTACTAGCAGTGAGACTCCAGGCATGCAATTTCTCTCTGT CCTTCAGTCCCTTCATCTCAAGGTTTAATTTAAATATGGTAACGCCTGTATGCAACTCCCAGCA TCCAGTAGGCACTCACTAAACACAGTTCTCCACCCTCCTTTTTTCCTCTGCCCCTCCCTCGG TTTTCCCACTACTTCCTGCATGGTGACACACCCATAGTTTGGAGCCATAAAACCCAACCCAG GTTGGACTCTCACCTCTCCAGCCCСTTCTGCTCCGGCCCTGTCCTCAAATTGGGGGGCTGA TGTCCCCATACACCTGGCTCTGGGTTCCCCTAACCCCAGAGTGCAGGACTAGGACCCGAGT GGACCTCAGGTCTGGCCAGGTCGCCATTGCCATGGAGACAGCAACAGTCCCCAGCCGCGG GTTCCCTAAGTGACTGGTTACTCTTTAACGTATCCACCCACCTTGGGTGATTAGAAGAATCAAT AAGATAACCGGGCGGTGGCAGCTGGCCGCACTCACCGCCTTCCTGGTGGACGGGCTCCTG GTGGCTGTGCTGCTGCTGTGAGCGGGCCCCTGCTCCTCCATGCCCCCAGCTCTCCGGCTG GGTGGGCTTAAGCTTCTCGACTTCCAGCTTGGCATAGAGGGTATATAATGGAAGCTCGACTT CCAGATCCGGTACTGTTGGTAAAGCCACCGGATCCAGCCACCATGGCCCAGTCCAAGCACG GCCTGACCAAGGAGATGACCATGAAGTACCGCATGGAGGGCTGCGTGGACGGCCACAAGT TCGTGATCACCGGCGAGGGCATCGGCTACCCCTTCAAGGGCAAGCAGGCCATCAACCTGTG CGTGGTGGAGGGCGGCCCCTTGCCCTTCGCCGAGGACATCTTGTCCGCCGCCTTCATGTA 
CGGCAACCGCGTGTTCACCGAGTACCCCCAGGACATCGTCGACTACTTCAAGAACTCCTGC CCCGCCGGCTACACCTGGGACCGCTCCTTCCTGTTCGAGGACGGCGCCGTGTGCATCTGC AACGCCGACATCACCGTGAGCGTGGAGGAGAACTGCATGTACCACGAGTCCAAGTTCTACG GCGTGAACTTCCCCGCCGACGGCCCCGTGATGAAGAAGATGACCGACAACTGGGAGCCCT CCTGCGAGAAGATCATCCCCGTGCCCAAGCAGGGCATCTTGAAGGGCGACGTGAGCATGTA CCTGCTGCTGAAGGACGGTGGCCGCTTGCGCTGCCAGTTCGACACCGTGTACAAGGCCAA GTCCGTGCCCCGCAAGATGCCCGACTGGCACTTCATCCAGCACAAGCTGACCCGCGAGGA CCGCAGCGACGCCAAGAACCAGAAGTGGCACCTGACCGAGCACGCCATCGCCTCCGGCTC CGCCTTGCCCGCCGCGCACCCGGGTTACTCTAGAGTCGGGGCGGCCGGCTAG 